\title{
HAUSDORFF SUMMABILITY METHODS
}

\author{
BY \\ B. E. RHOADES
}

1. Introduction. In order to make this paper somewhat self-contained, and, because there appears to be very little agreement on notation by authors in this field, I shall define most of the terminology used, and give specific references to concepts which are used but not defined herein.

The first grouping of statements is applicable to infinite matrices in general. Let $x$ denote a sequence, $A$ an infinite matrix for which $A_{n}(x)=\sum_{k} a_{n k} x_{k}$ is defined. The convergence domain of a matrix $A$, denoted by $(A)$, consists of the set of sequences $x$ such that $\left\{A_{n}(x)\right\}$ is convergent. Two matrices are said to be equivalent if they both have the same convergence domain. A matrix $A$ is called conservative if (i) $a_{k}=\lim _{n} a_{n k}$ exists for each $k$, (ii) $t=\lim _{n} \sum_{k} a_{n k}$ exists, and (iii) $A$ has finite norm, where $\|A\|$ $=\sup _{n} \sum_{k}\left|A_{n k}\right|$. A matrix $A$ is said to be regular if $A$ is limit preserving; i.e., for every convergent sequence $x$ with limit $l, A_{n}(x) \rightarrow l$. Necessary and sufficient conditions on a matrix $A$ for regularity are: (i) $a_{k}=0$ for each $k$, (ii) $t=1$, and (iii) $A$ has finite norm. A matrix $A$ is said to be totally regular, if $A$ is regular, and if, for every sequence $x$ such that $x_{n} \rightarrow+\infty, A_{n}(x) \rightarrow+\infty$. If a matrix $A$ possesses a two-sided inverse, the statement $B$ t.s. $A$ is equivalent to the statement that $B A^{-1}$ is totally regular. For two matrix methods $A$ and $B, B$ is said to be totally stronger than $A$, written $B$ t.s. $A$, if for each sequence $x$ for which $A_{n}(x) \rightarrow l, B_{n}(x) \rightarrow l,|l| \leqq \infty . B$ is not totally stronger than $A$, written $B$ n.t.s. $A$, if $A_{n}(x) \rightarrow l$ implies $B_{n}(x) \rightarrow l, l$ finite, but there exists a sequence $x$ for which $A_{n}(x) \rightarrow+\infty$ and $B_{n}(x) \rightarrow+\infty$. Two matrices are said to be totally equivalent if $A$ t.s. $B$ and $B$ t.s. $A$. A matrix $A$ is called triangular if all the elements above the main diagonal are zero.

Let $\mu$ denote a sequence. Define a linear difference operator $\Delta$, operating on $\mu$, by $\Delta \mu_{k}=\mu_{k}-\mu_{k+1}, \Delta^{n} \mu_{k}=\Delta\left(\Delta^{n-1} \mu_{k}\right)=\sum_{j}(-1)^{i} C_{n, j} \mu_{k+j}$. A Hausdorff matrix $H=\left(h_{n k}\right)$ is a triangular matrix defined by $h_{n k}=C_{n, k} \Delta^{n-k} \mu_{k}$. Hence $\mu$ is called the generating sequence. A sequence $\mu$ is called totally monotone if all of the successive differences are non-negative; i.e., $\Delta^{n} \mu_{k} \geqq 0 ; k, n=0,1,2, \cdots$. It should be noted that the row and column indexing for Hausdorff matrices starts with 0 instead of 1.

A Hausdorff matrix has finite norm if and only if the generating sequence can be expressed as the difference of two totally monotone sequences. Every Hausdorff matrix with finite norm is conservative and enjoys the additional properties that $\sum_{k} h_{n k}=\mu_{0}$ for each $n$, hence $t=\mu_{0}, \lim _{n} h_{n k}=0$ for each positive $k$, and $h_{0}=\lim _{n} h_{n, 0}$ exists. Thus the regularity conditions for a Hausdorff

Received by the editors March 14, 1961. 
matrix can be described by (i) $h_{0}=0$, (ii) $\mu_{0}=1$, and (iii) $H$ has finite norm. A Hausdorff matrix is totally regular if and only if it is regular, and the moment generating sequence is totally monotone.

In speaking of a Hausdorff matrix $H$ generated by a sequence $\mu$, I shall denote the matrix by $H_{\mu}$, the method by $H \sim \mu$, and the convergence domain of $H_{\mu}$ by $(H, \mu)$, except that I shall denote the convergence domain of the Cesàro matrix of order 1 by $(C, 1)$. For other basic properties of a Hausdorff matrix see [6]. The identity matrix shall be denoted by $I$.

It is a well-known result $[1$, p. 452$]$ that two regular Hausdorff methods with nonvanishing moment sequences cannot be totally equivalent unless they are identical. Hence the concept of totally stronger serves as a partial ordering of equivalent Hausdorff methods.

Basu in [1] compares totally the Cesàro matrices, usually denoted by $C^{\alpha}$ or $C_{\alpha}$, and the Hölder matrices denoted by $H^{\alpha}$ or $H_{\alpha}$. In [4] he compares totally the $H^{\alpha}$ and $\Gamma_{a}^{\alpha}$ matrices, where $\Gamma_{a}^{\alpha}$, called the Gamma matrix, has $[a /(n+a)]^{\alpha}$ as a generating sequence, $a, \alpha>0$. In [11] the author compares totally the Gamma and Cesàro methods, using the results of $[1 ; 4]$ to obtain the following facts.

Let $0<a, a^{\prime}<1, b>1$. Then,

$\mathrm{R}(\mathrm{i})$ for $0<a \leqq(\alpha+1) / 2<1+\alpha \leqq a^{\prime}<1, \Gamma_{b}^{\alpha}$ t.s. $H_{\alpha}$ t.s. $\Gamma_{a^{\prime}}^{\alpha}$ t.s. $C_{\alpha}$ t.s. $\Gamma_{a}^{\alpha}$, but not conversely. and

R(ii) for $0<a \leqq(\alpha+1) / 2<1, \Gamma_{a}^{\alpha}$ t.s. $C_{\alpha}$ t.s. $H_{\alpha}$ t.s. $\Gamma_{b}^{\alpha}$, but not conversely;

$\mathrm{R}$ (iii) for $\alpha>1,2 b \geqq \alpha+1, \Gamma_{a}^{\alpha}$ t.s. $H_{\alpha}$ t.s. $C_{\alpha}$ t.s. $\Gamma_{b}^{\alpha}$, but not conversely.

Basu in [2] compares totally the hypergeometric and Cesàro methods. In $\$ 3$ of this paper the hypergeometric method is compared totally with the Gamma method, taking advantage of the above facts and the results of [2]. The proofs employ a technique believed to be new. The basic properties of this technique are developed in $\$ 2$.

In $\$ 4$ the $C_{1}$ and $\Gamma_{a}^{1}$ methods are compared totally with three methods defined and discussed by Greenberg and Wall (hereafter referred to as $G$ and W) in [5, pp. 780-783].

In $\$ 5$, the equivalence is established of certain Hausdorff methods for complex sequences, whose analogues for the corresponding real sequences are well known.

In $\$ 6$ a Hausdorff method of summability is defined. This method of summability, involving two parameters, is shown to be equivalent to $C^{\alpha}$ for nonnegative $\alpha$, and reduces to the Hölder or Gamma methods of order $\alpha$, for proper choice of parameters.

In $\$ 7$ this method is compared totally with the Cesàro, Hölder, and Gamma methods.

$\$ 8$ generalizes some results of $[13]$. 


\section{Products of Hausdorff matrices.}

THEOREM 2.1. Let $\mu=\left\{\mu_{n}\right\}$ be a totally monotone sequence with at least two nonzero elements. Then $\mu_{n} \neq 0$ for any $n$.

Proof. Assume there exists an integer $k$ such that $\mu_{k-1} \neq 0$ and $\mu_{k}=0$. Then, either $\mu_{n}=0$ for all $n \geqq k$ or else there exists at least one integer $m>k$ such that $\mu_{m} \neq 0$. However, the latter condition implies $\mu$ is not totally monotone. Hence, $\mu_{n}=0$ for all $n \geqq k$. Then $\Delta^{r} \mu_{k}=\mu_{k}$ for $r=0,1,2, \cdots$, and the associated Hausdorff matrix $H_{\mu}=\left(h_{r k}\right)$ has the property that $\lim _{r \rightarrow \infty} h_{r k}=\infty$, and hence $H$ is not conservative. But $\mu$ totally monotone implies $H_{\mu}$ is conservative. Therefore $\mu$ is not totally monotone.

Theorem 1 cannot be weakened, since the sequence $\{1,0,0, \cdots\}$ is totally monotone.

However, sequences of the form $\{c, 0,0, \cdots\}, c$ a constant, form the set of co-null (see $[14$, p. 61$]$ for the definition of co-null) Hausdorff matrices, which are not regular. Hence it is true that every totally regular Hausdorff method has a nonvanishing moment sequence.

A triangular matrix is called a triangle if there are no zero elements on the main diagonal. The discussion following Theorem 2.1 implies that every totally regular Hausdorff matrix $H$ is a triangle. Hence, $H^{-1}$ always exists, which leads to the following theorem, true for triangles in general.

Theorem 2.2. Let $A$ and $B$ be triangles. If $A$ t.s. $B$ and $B$ is totally regular, then $A$ is totally regular.

Proof. $A$ t.s. $B$ iff $A B^{-1}$ is totally regular. $\left(A B^{-1}\right)(B)=A$. Therefore $A$ is totally regular, since it is the product of two totally regular methods.

Since the product of two totally regular sequences is also totally regular (see, e.g. [3, p. 393, Lemma 4]), a feasible problem is to compare the total strength of the product with respect to the total strength of either.

THEOREм 2.3. Let $H \sim \mu_{1}, H \sim \mu_{2}$ be totally regular Hausdorff methods. Then $H \sim \mu_{1} \mu_{2}$ t.s. $H \sim \mu_{1}, H \sim \mu_{2}$.

The proof is trivial since $H_{\mu_{1} \mu_{2}} / H_{\mu_{k}}=H_{\mu_{j}}, j, k=1,2, j \neq k$, which is totally regular.

This theorem enables us to give new proofs of known results. For example, it can be used to prove $H_{\beta}$ t.s. $H_{\alpha}$ for $\beta>\alpha>0$. Let $\mu_{1_{k}}=(k+1)^{-(\beta-\alpha)}, \mu_{2_{k}}$ $=(k+1)^{-\alpha}$. Then $\mu_{1_{k}} \mu_{2_{k}}=(k+1)^{-\beta}$, and the result follows by Theorem 2.3.

Similarly, we can show that for $0<\alpha<\beta, \Gamma_{a}^{\beta}$ t.s. $\Gamma_{a}^{\alpha}$.

However, Theorem 2.3 has other uses, which will soon be demonstrated.

THEOREM 2.4. Let $H \sim \mu_{1}, H \sim \mu_{2}$ be two Hausdorff methods with nonvanishing moment sequences. Then $H \sim \mu_{1}$ t.s. $H \sim \mu_{2} \leftrightarrow H \sim \mu_{2}^{-1}$ t.s. $H \sim \mu_{1}^{-1}$.

Proof. Let $\lambda_{k}=\mu_{1_{k}} / \mu_{2_{k}}=\left(\mu_{2_{k}}\right)^{-1} /\left(\mu_{1_{k}}\right)^{-1}$. Q.E.D., since $\lambda$ is given to be totally regular. 
TheOREM 2.5. Let $H \sim \mu_{i}, H \sim \lambda_{i}$ be Hausdorff methods such that $H \sim \mu_{i}$ t.s. $H \sim \lambda_{i}, \lambda_{i}$ nonvanishing $i=1$, 2. Then $H \sim \mu_{1} \mu_{2}$ t.s. $H \sim \lambda_{1} \lambda_{2}$.

Proof. Let $\alpha_{k}=\mu_{1_{k}} \mu_{2_{k}} / \lambda_{1_{k}} \lambda_{2_{k}}=\left(\mu_{1_{k}} / \lambda_{1_{k}}\right) \cdot\left(\mu_{2_{k}} / \lambda_{2_{k}}\right)$. Therefore $H \sim \alpha$ is totally regular, since it is the product of two totally regular methods.

Theorem 2.6. Let $|\alpha|<1$. Then $C_{a}^{-1}$ t.s. $C_{-\alpha}$.

Proof. For $\alpha \geqq 0$, an outline of a proof is contained in [10], and will be omitted here. For $\alpha<0$, use the result of $[10]$ in conjunction with Theorem 2.4.

Note that Theorem 2.6 together with Theorem 2.2 states that $C_{\gamma}^{-1}$ is totally regular for $-1<\gamma<0$.

Corollary 2.1. Let $0<\gamma<1$. Then $H_{-\gamma}$ t.s. $C_{\gamma}^{-1}$.

Proof. From R(ii), $C_{\gamma}$ t.s. $H_{\gamma}$. From Theorem $2.4 H_{\gamma}^{-1}=H_{-\gamma}$ t.s. $C_{\gamma}^{-1}$.

Corollary 2.2. For $0<1-a \leqq \gamma<1, C_{-\gamma}^{-1}$ t.s. $\Gamma_{a}^{\gamma}$.

Proof. By (i), $\Gamma_{a}^{-\gamma}$ t.s. $C_{-\gamma}$ for $0<1-\gamma \leqq a<1$, i.e., $1>\gamma \geqq 1-a>0$. Use Theorem 2.4.

CoRollary 2.3. For $0<\gamma \leqq 1-2 a<1, \Gamma_{a}^{\gamma}$ t.s. $C_{-\gamma}^{-1}$.

The proof is the same as that of Corollary 2.2 and will be omitted.

Theorem 2.4 together with Theorem 2.6 is very useful in comparing the relative strength of Cesàro-Hölder type methods as $\alpha$ changes from positive to negative values, $|\alpha|<1$.

For example, from (ii), $C_{\alpha}$ t.s. $H_{\alpha}$ for $0<\alpha<1$. From Theorem $2.4\left(H_{\alpha}\right)^{-1}$ $=H_{-\alpha}$ t.s. $C_{\alpha}^{-1}$. But, from Theorem $2.6 C_{\alpha}^{-1}$ t.s. $C_{-\alpha}$. Thus $H_{\gamma}$ t.s. $C_{\gamma}$ for $\gamma=-\alpha$, yielding a new proof of $[1$, Theorem 1 (i) $]$.

Theorem 1 of $[2$, pp. 229-233] states that, for $\alpha, \beta, \alpha+\beta>-1$,

$$
C_{\alpha} C_{\beta} \text { t.s. } C_{\alpha+\beta} \text {, }
$$

$\alpha \beta>0$,

B(ii)

$$
C_{\alpha+\beta} \text { t.s. } C_{\alpha} C_{\beta} \text {, }
$$$$
\alpha \beta<0 \text {. }
$$

Assuming the truth of $B(i)$ it is possible to give an independent proof of B(ii). Consider $C_{\alpha}^{-1} C_{\beta}^{-1} C_{\alpha+\beta}, \alpha \beta<0$. Then either $-1<\alpha<0$ or $-1<\beta<0$. Since the above expression is symmetric in $\alpha$ and $\beta$, it will be sufficient to prove the result for $-1<\alpha<0$.

Since $\alpha \beta<0, \beta>0$, we have two possibilities. Either $\alpha+\beta \geqq 0$ or $\alpha+\beta<0$.

CASE I. Assume $\alpha+\beta \geqq 0$.

By Theorems 2.5 and 2.6, $C_{\alpha}^{-1} C_{\beta}^{-1} C_{\alpha+\beta}$ t.s. $C_{-\alpha} C_{\beta}^{-1} C_{\alpha+\beta}$. From B(i), $C_{-\alpha} C_{\alpha+\beta}$ t.s. $C_{-\alpha+\alpha+\beta}=C_{\beta}$. Using Theorem $2.5 C_{-\alpha} C_{\beta}^{-1} C_{\alpha+\beta}$ t.s. $C_{\beta} C_{\beta}^{-1}=I$. But $I$ is totally regular. Therefore, from Theorem $2.2 C_{\alpha}^{-1} C_{\beta}^{-1} C_{\alpha+\beta}$ is totally regular; i.e., $C_{\alpha+\beta}$ t.s. $C_{\alpha} C_{\beta}$.

CAsE II. Assume $\alpha+\beta<0$. Then $0<\beta<1$. From Theorems 2.5 and 2.6, 
$C_{\alpha}^{-1} C_{\beta}^{-1} C_{\alpha+\beta}$ t.s. $C_{\alpha}^{-1} C_{-\beta} C_{\alpha+\beta}$. From B(i), $C_{-\beta} C_{\alpha+\beta}$ t.s. $C_{-\beta+\alpha+\beta}=C_{\alpha}$. Hence, by Theorem 2.5, $C_{\alpha}^{-1} C_{\beta}^{-1} C_{\alpha+\beta}$ t.s. $C_{\alpha}^{-1} C_{\alpha}=I$. I is totally regular. Hence, by Theorem 2.2, $C_{\alpha}^{-1} C_{\beta}^{-1} C_{\alpha+\beta}$ is totally regular.

3. Comparison of the hypergeometric method with the $\Gamma_{a}^{\alpha}$ method. For the sequence which defines the hypergeometric method $(H, \alpha, \beta, \gamma)$, see $[2$, p. 227].

As Basu [2] points out, the hypergeometric method becomes more workable when it is expressed in terms of its Cesàro equivalent; i.e. $(H, \alpha, \beta, \gamma)$ $=C_{\alpha-1}^{-1} C_{\beta-1}^{-1} C_{\gamma-1}$.

ThEOREM 3.1. For $0<\alpha, \beta \leqq 1, \beta<1+\gamma \leqq 1$ or $\alpha \leqq 1+\gamma \leqq 1,(H, \alpha, \beta, \gamma+1)$ t.s. $\Gamma_{b}^{\gamma}, b>1$.

Proof. Let $H=C_{\alpha-1}^{-1} C_{\beta-1}^{-1} C_{\gamma} \Gamma_{b}^{-\gamma}$. Since $\beta \leqq 1+\gamma ; C_{\gamma}$ t.s. $C_{\beta-1}$; i.e., $C_{\gamma} C_{\beta-1}^{-1}$ is totally regular. But $C_{\alpha-1}^{-1}$ and $\Gamma_{b}^{-\gamma}$ are also totally regular. Therefore $H$ is totally regular. It is clear that the roles of $\alpha$ and $\beta$ are interchangeable in the above argument.

Theorem 3.2. For $\alpha, \beta, b>1,0 \leqq \gamma \leqq \alpha-1$ or $0 \leqq \gamma \leqq \beta-1$, $\Gamma_{b}^{\gamma}$ t.s. $(H, \alpha, \beta, \gamma+1)$.

Proof. Let $H=C_{\alpha-1} C_{\beta-1} C_{\gamma}^{-1} \Gamma_{b}^{\gamma}$. Since $\alpha-1 \geqq \gamma, C_{\alpha-1} C_{\gamma}^{-1}$ is totally regular. So also are $\Gamma_{b}^{\gamma}$ and $C_{\beta-1}$. Therefore $H$ is totally regular. As before, $\alpha$ and $\beta$ are interchangeable.

THEOREM 3.3. For $0 \leqq \alpha, \beta, \gamma<1, \alpha \leqq 1-\gamma \leqq a<1,(H, \alpha, \beta, \gamma+1)$ t.s. $\Gamma_{a}^{\gamma}$.

Proof. Let $H=C_{\alpha-1}^{-1} C_{\beta-1}^{-1} C_{\gamma} \Gamma_{a}^{-\gamma}=C_{\alpha-1}^{-1} C_{\beta-1}^{-1} C_{\gamma} \Gamma_{a}^{-\gamma} C_{-\gamma}^{-1} C_{-\gamma}$. From (i) $\Gamma_{a}^{-\gamma}$ t.s. $C_{-\gamma}$. By Theorem $2.4, C_{-\gamma}^{-1}$ t.s. $\Gamma_{a}^{\gamma}$; i.e., $C_{-\gamma}^{-1} \Gamma_{a}^{-\gamma}$ is totally regular. Since $1-\gamma \geqq \alpha, C_{-\gamma} C_{\alpha-1}^{-1}$ is totally regular. So also are $C_{\gamma}$ and $C_{\beta-1}^{-1}$. Therefore $H$ is totally regular.

By extremely lengthy calculations it can be shown that, for $0 \leqq \alpha, \beta, \gamma<1$, $a \geqq \gamma, \beta,(H, \alpha, \beta, \gamma+1)$ t.s. $\Gamma_{a}^{\gamma}$.

Basu [2, p. 228] shows that $C_{\gamma}$ t.s. $(H, \alpha, \beta, \gamma+1)$ for $\alpha, \beta \geqq 1, \gamma>-1$, and that $(H, \alpha, \beta, \gamma+1)$ t.s. $C_{\gamma}$ for $0<\alpha, \beta \leqq 1, \gamma>-1$. Using these results in conjunction with Theorems 2.6, 3.1-3.3, Corollaries 2.1-2.3, and R(i)-R(iii), we have the following table. The notation "t.s." has been replaced by an arrow pointing to the weaker method.

Let $0<a, a^{\prime}<1, b \geqq 1,0<\alpha_{1}, \beta_{1} \leqq 1, \alpha_{2}, \beta_{2} \geqq 1$.

For $-1<\gamma \leqq 0$,

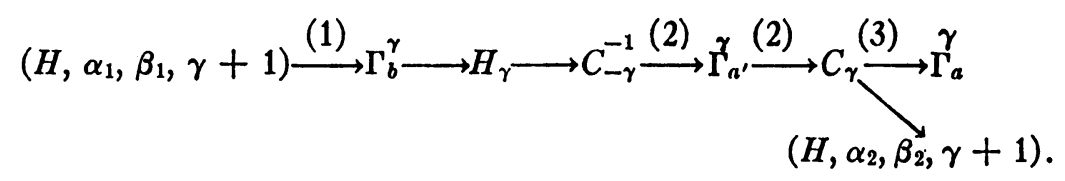

For $0 \leqq \gamma<1$, 


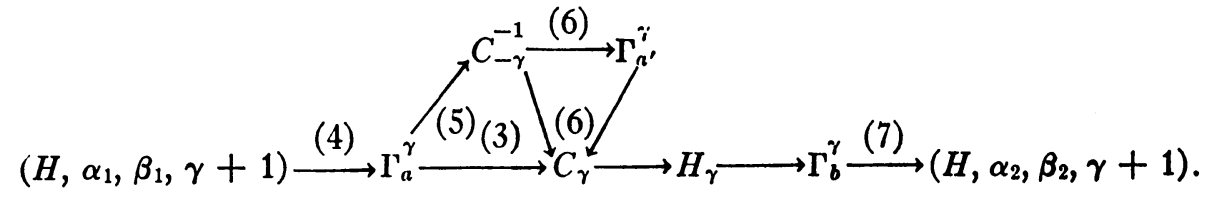

For $\gamma>1$,

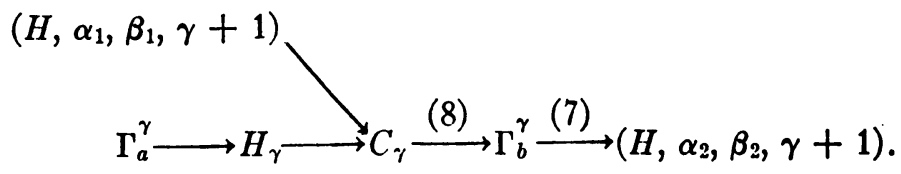

(1) $\beta_{1} \leqq 1+\gamma \leqq 1$, or $\alpha_{1} \leqq 1+\gamma \leqq 1$. (5) $0<\gamma \leqq 1-2 a<1$.

(2) $0<1+\gamma \leqq a<1$.

(6) $0<1-a^{\prime} \leqq \gamma<1$.

(3) $0<a \leqq(\gamma+1) / 2<1$.

(7) $0 \leqq \gamma \leqq \alpha_{2}-1$, or $0 \leqq \gamma \leqq \beta_{2}-1$.

(4) $\alpha_{1} \leqq 1-\gamma \leqq a<1$.

(8) $2 b \geqq \gamma+1$.

I conclude the discussion of hypergeometric methods with the following four negative results.

Theorem 3.4. For $-1<\gamma \leqq 0, \alpha, \beta<1, c>0, \Gamma_{c}^{\gamma}$ n.t.s. $(H, \alpha, \beta, \gamma+1)$.

Proof. Consider $C_{\alpha-1} C_{\beta-1} C_{\gamma}^{-1} \Gamma_{c}^{\gamma}$. Then $\mu_{n}=\delta_{n} \lambda_{n} v_{n}$, where

$$
\begin{aligned}
& \delta_{n}=\frac{\Gamma(n+1) \Gamma(\alpha)}{\Gamma(n+\alpha)}, \quad \lambda_{n}=\frac{\Gamma(n+1) \Gamma(\beta)}{\Gamma(n+\beta)}, \\
& v_{n}=\frac{\Gamma(n+\gamma+1)}{\Gamma(n+1) \Gamma(\gamma+1)} \cdot \frac{c^{\gamma}}{(n+c)^{\gamma}} .
\end{aligned}
$$

A necessary condition for $H_{\mu}$ to be conservative is that $\mu$ be a convergent sequence. Since $\Gamma_{c}^{\gamma}$ and $C_{\gamma}$ are equivalent for all $\gamma>-1, \gamma$ is a non-null convergent sequence. Let $\nabla=\min v_{n}$. Since $\delta_{n}, \lambda_{n}$ are positive, $\mu_{n} \geqq \delta_{n} \lambda_{n} \nabla$. For $\alpha, \beta<1, \delta_{n} \rightarrow \infty$ and $\lambda_{n} \rightarrow \infty$. Therefore $\mu$ is not convergent and $H_{\mu}$ is not conservative, hence not totally regular.

TheOREM 3.5. For $\gamma>0, \alpha, \beta>1, b>1,(H, \alpha, \beta, \gamma+1)$ n.t.s. $\Gamma_{b}^{\gamma}$.

Proof. Let $H_{\mu}^{-1}=C_{\alpha-1}^{-1} C_{\beta-1}^{-1} C_{\gamma} \Gamma_{b}^{-\gamma}$. Then $\mu_{n}^{-1}=\delta_{n}^{-1} \lambda_{n}^{-1} v_{n}^{-1}$ where $\delta_{n}, \lambda_{n}, v_{n}$ are defined as in Theorem 3.4 with $c=b$. As before, $v^{-1}$ is non-null and convergent. Let $\nabla^{-1}=\min v_{n}^{-1}$. Then $\mu_{n}^{-1} \geqq \delta_{n}^{-1} \lambda_{n}^{-1} \mathbf{v}^{-1}$. Since $\alpha, \beta>1, \delta_{n}^{-1} \rightarrow \infty$ and $\lambda_{n}^{-1} \rightarrow \infty$. Therefore $\mu^{-1}$ is not convergent.

Theorem 3.6. For $\gamma \geqq 0,0<\alpha, \beta \leqq 1, c>0, \Gamma_{c}^{\gamma}$ n.t.s. $(H, \alpha, \beta, \gamma+1)$.

Proof. Let $\mu_{n}=\delta_{n} \lambda_{n} v_{n}$, where $\delta_{n}, \lambda_{n}, v_{n}$ are as defined in Theorem 3.4. 
Again $\nabla>0$, where $\nabla=\min v_{n}$. Then $\mu_{n} \geqq \delta_{n} \lambda_{n} \nabla$. But $\delta_{n} \rightarrow \infty$ and $\lambda_{n} \rightarrow \infty$, since $\alpha, \beta<1$. Therefore $\mu$ is not convergent.

TheOREM 3.7. For $\gamma \geqq 1,0<\alpha, \beta \leqq 1, H_{\gamma}$ n.t.s. $(H, \alpha, \beta, \gamma+1)$.

Proof. Assume $H_{\gamma}$ t.s. $(H, \alpha, \beta, \gamma+1)$. From (iii), $\Gamma_{a}^{\gamma}$ t.s. $H_{\gamma}$ for $0<a<1$. Hence $\Gamma_{a}^{\gamma}$ t.s. $(H, \alpha, \beta, \gamma+1)$, contradicting Theorem 3.6.

Theorem 3.7 can also be proved by using a technique similar to that employed in the proof of Theorem 3.4.

The following are some open questions.

(i) For $-1<\gamma \leqq 0,0<\alpha_{1}, \beta_{1} \leqq 1, b>1, \quad \beta_{1}>1+\gamma$ and $\alpha_{1}>1+\gamma$, is $\left(H, \alpha_{1}, \beta_{1}, \gamma+1\right)$ t.s. $\Gamma_{b}^{\gamma}$ ?

(ii) For $0<\gamma<1,0<\alpha_{1}, \beta_{1} \leqq 1,0<a<1, a<\beta_{1}$ or $a<\gamma$, is $\left(H, \alpha_{1}, \beta_{1}, \gamma+1\right)$ t.s. $\Gamma_{a}^{\gamma}$ ?

For $\gamma>1,0<a<1,0<\alpha_{1}, \beta_{1} \leqq 1$, is

(iii) $\left(H, \alpha_{1}, \beta_{1}, \gamma+1\right)$ t.s. $\Gamma_{a}^{\gamma}$ ?

(iv) $\left(H, \alpha_{1}, \beta_{1}, \gamma+1\right)$ t.s. $H_{\gamma}$ ?

4. Total comparison among three Hausdorff methods and the $C_{1}$ method. Throughout this section, $a, b, c$ are real positive numbers with $c$ arbitrary, $0<a \leqq 1, b>1$.

Combining the relations (iii) and (iv) of $[2$, p. 228], or stating Theorem 5 of [11], gives the following result, which is stated here for ready reference.

R5

$$
\Gamma_{a}^{1} \text { t.s. } C_{1} \text { t.s. } \Gamma_{b}^{1} \text {. }
$$

The three Hausdorff methods from $G$ and $W$ shall be denoted by $H \sim \alpha_{1}(t, k, c), H \sim \alpha_{2}(t, r)$, and $H \sim \alpha_{3}(t, r)$, where

$$
\alpha_{1}(t, k, c)=\frac{1-\alpha(t, k, c)}{c_{1} t}, \quad \alpha(t, k, c)=c^{k}(t+c)^{-k},
$$

$k=1,2,3, \cdots, c>0, c_{1}$ a constant such that $\alpha_{1}(0, k, c)=1$;

$$
\begin{aligned}
& \alpha_{2}(t, r)=\frac{1-r^{t}}{t \log \frac{1}{r}}=\int_{0}^{1} u^{t} d \phi_{2}(u), \quad 0<r<1, \\
& \phi_{2}(u)=\left\{\begin{array}{cc}
0, & 0 \leqq u \leqq r, \\
1-\frac{\log u}{\log r}, & r<u \leqq 1,
\end{array}\right.
\end{aligned}
$$

and

$$
\alpha_{s}(l, r)=\frac{1-r^{t+1}}{(t+1)(1-r)}=\int_{0}^{1} u^{i} d \phi_{3}(u), \quad 0<r<1,
$$




$$
\phi_{3}(u)=\left\{\begin{array}{c}
0 \\
\frac{u-r}{1-r}
\end{array}\right.
$$

First consider $\alpha_{1}(t, k, c)$, and observe that it can be written as $c_{1}^{-1} \sum_{r=1}^{k} c^{r-1}(t+c)^{-r}$. Hence $c_{1}=k / c$.

Since $\alpha_{1}(t, k, c)=k^{-1} \sum_{r=1}^{k} c^{r}(t+c)^{-r}, H \sim \alpha_{1}(t, k, c)$ is totally regular, since it is a finite sum of totally regular methods with positive coefficients, and $\alpha_{1}(0, k, c)=1$.

Theorem 4.1. $H \sim \alpha_{1}(t, k, c)$ t.s. $\Gamma_{c}^{1}$ for $k=1,2, \cdots$.

Since ordinary equivalence has been established by $\mathrm{G}$ and $\mathrm{W}$ for $H \sim \alpha(t, \alpha, c)$ and $C_{1}$, and since $C_{1}$ and $\Gamma_{c}^{1}$ are equivalent, Theorem 4.1 is proved, as are all of the positive results stated here, by showing that the moment generating sequence is totally monotone, or is a finite product of totally monotone sequences.

Corollary 4.1. $H \sim \alpha_{1}(t, k, a)$ t.s. $C_{1}$.

This result follows from Theorem 4.1 with $c=a$, and R5.

THEOREM 4.2.

(i) $(C, 1)=\left(H, \alpha_{1}(t, k, c)\right), k=1,2,3, \cdots$.

(ii) $C_{1}$ n.t.s. $H \sim \alpha_{1}(t, k, b), k=2,3,4, \cdots$.

(iii) $H \sim \alpha_{1}(t, k, b)$ n.t.s. $C_{1}, k=2,3,4, \cdots$.

(iv) $C_{1}$ t.s. $H \sim \alpha_{1}(t, 1, b)$.

Part (i) has been established by $\mathrm{G}$ and $\mathrm{W}$.

Part (ii) of Theorem 4.2, and most of the negative results of this section are proved by showing that the moment generating function is not totally monotone. A moment generating function is a continuously differentiable function $f(t)$, whose values on the non-negative integers are the terms of a corresponding moment sequence $\mu$; i.e., $f(n)=\mu_{n}$. The sequence $\mu$ is totally monotone if and only if $(-1)^{n} f^{(n)}(t) \geqq 0$ for $t>0, n=0,1,2, \cdots$, and $f(0+)$ $\leqq f(0)$.

Let $\mu(t)=\alpha_{1}(t, k, b) \cdot(t+1)=\mu_{1}(t) \cdot \mu_{2}(t)$, where $\mu_{1}(t)=t+1, \mu_{2}(t)=\alpha_{1}(t, k, b)$ $=k^{-1} \sum_{r=1}^{k}[b /(t+b)] r . \mu_{1}^{\prime}(t)=1, \mu_{1}^{(n)}(t)=0, n>1$.

$$
\mu_{2}^{(n)}(t)=(-1)^{n} k^{-1} \sum_{r=1}^{k} r(r+1) \cdots(r+n-1) b^{r}(t+b)^{-r-n},
$$

$n=1,2,3, \cdots$.

$$
\mu^{(n)}(t)=\sum_{j=0}^{n}\left(\begin{array}{l}
n \\
j
\end{array}\right) D_{t}^{n-j}\left[\mu_{1}(t)\right] \cdot D_{t}^{j}\left[\mu_{2}(t)\right]=\sum_{j=n-1}^{n}\left(\begin{array}{l}
n \\
j
\end{array}\right) \mu_{1}^{(n-j)}(t) \cdot \mu_{2}^{(j)}(t) .
$$




$$
\begin{aligned}
\mu^{(n)}(t)= & \frac{n(-1)^{n-1} \sum_{r=1}^{k} \frac{r(r+1) \cdots(r+n-2) b^{r}}{k}}{(t+b)^{r+n-1}} \\
& +\frac{(-1)^{n}(t+1)}{k} \sum_{r=1}^{k} \frac{r(r+1) \cdots(r+n-1) b^{r}}{(t+b)^{r+n}} . \\
(-1)^{n} \mu^{(n)}(t)= & \frac{1}{k} \sum_{r=1}^{k} \frac{r(r+1) \cdots(r+n-2) b^{r}}{(t+b)^{r+n-1}}\left\{\frac{(t+1)(r+n-1)}{(t+b)}-n\right\} .
\end{aligned}
$$

Let $f(t)=(t+1)(r+n-1)-n(t+b)=(r-1) t+r-1+n(1+b)$.

For $r=1, f(t)<0$ for $t \geqq 0, n=1,2,3, \cdots$.

For $r>1, f(t)=(r-1)[t+1-(b-1) n /(r-1)]$. Fix $t=t_{0}$. For $n$ sufficiently large, $f\left(t_{0}\right)<0$. Therefore $\mu(t)$ is not totally monotone.

Proof of (iii). Let

$1 / \mu(t)=\frac{1}{t+1} \cdot \frac{c_{1} t}{1-[b /(t+b)]^{k}} \cdot$

$D_{t}(1 / \mu(t))$

$$
=c_{1}\left\{\frac{(t+1)\left[1-b^{k} /(t+b)^{k}\right]-t\left[1-\left(\frac{b}{t+b}\right)^{k}+\frac{(t+1) k b^{k}}{(t+b)^{k+1}}\right]}{(t+1)^{2}\left[1-b^{k} /(t+b)^{k}\right]^{2}}\right\} .
$$

$(-1) D_{t}(1 / \mu(t))=\frac{c_{1} f(t)}{(t+1)^{2}\left[1-b^{k} /(t+b)^{k}\right]^{2}}$,

where

$$
\begin{aligned}
f(t) & =\frac{b^{k}}{(t+b)^{k}}\left\{\frac{k t(t+1)}{t+b}+1\right\}-1 \\
& =\frac{b^{k}}{(t+b)^{k+1}}\left(k t^{2}+k t+t+b\right)-1 .
\end{aligned}
$$

Since $k \geqq 2 \lim _{t \rightarrow \infty} f(t)=-1$. Therefore there exists a value $t_{1}>0$ such that $f(t)<0$ for $t>t_{1}$. Therefore $1 / \mu(t)$ is not totally monotone.

Proof of (iv). $H \sim \alpha_{1}(t, 1, b)=\Gamma_{b}^{1}$. Q.E.D. by R5.

THEOREM 4.3. $H \sim \alpha_{2}(t, r)$ n.t.s. $\Gamma_{c}^{1}$.

Proof. It shall be sufficient to show that $\lambda(t)$ is not totally monotone, where $\lambda(t)=\left(1-r^{t}\right)(t+c) / t$.

Writing $r^{t}$ as $\left(e^{\log r}\right)^{t}$, and using the infinite series expansion for $e^{x}$, we obtain 


$$
\begin{aligned}
\lambda(t) & =-\left(1+\frac{c}{t}\right) \sum_{k=1}^{\infty} \frac{(t \log r)^{k}}{k !} \\
& =-\sum_{k=1}^{\infty} \frac{(t \log r)^{k}}{k !}-c \sum_{k=1}^{\infty} \frac{(\log r)^{k} t^{k-1}}{k !} \\
& =-c \log r-\sum_{k=1}^{\infty} \frac{(t \log r)^{k}(k+1+c \log r)}{(k+1) !}
\end{aligned}
$$

For $t$ positive and sufficiently small, the sign of $\lambda^{(k)}(t)$ will be the same as that of the coefficient $c_{k}$ of $t^{k}$. From the above expression for $\lambda(t)$ we see that

$$
c_{k}=\frac{(-1)^{k+1}(|\log r|)^{k}}{(k+1) !}(k+1+c \log r) .
$$

For each $r, 0<r<1, k+1+c \log r>0$ for all $k$ sufficiently large. Therefore $c_{k}$ is negative for all $k$ sufficiently large, and positive $t$, sufficiently small, and $\lambda(t)$ is not totally monotone.

Corollary 4.2. $H \sim \alpha_{2}(t, r)$ n.t.s. $C_{1}$.

If $H \sim \alpha_{2}(t, r)$ t.s. $C_{1}$, then, from R5, $H \sim \alpha_{2}(t, r)$ t.s. $\Gamma_{b}^{1}$, contradicting Theorem 4.3.

Corollary 4.3. $H \sim \alpha_{2}(t, r)$ n.t.s. $H \sim \alpha_{1}(t, k, c)$.

If $H \sim \alpha_{2}(t, r)$ t.s. $H \sim \alpha_{1}(t, k, c)$, using Theorem 4.1 gives a contradiction to Theorem 4.3.

Theorem 4.4. $C_{1}$ t.s. $H \sim \alpha_{2}(t, r)$ is not possible.

From $\mathrm{G}$ and $\mathrm{W},\left(H, \alpha_{2}(t, r)\right)_{\neq}^{\supset}(C, 1)$.

THEOREM 4.5. $H \sim \alpha_{1}(t, k, c)$ n.t.s. $H \sim \alpha_{2}(t, r)$.

Let

$$
\mu_{n}=\frac{1-\left(\frac{c}{n+c}\right)^{k}}{\frac{k n}{c}} \cdot \frac{n \log \frac{1}{r}}{1-r^{n}}=\frac{c \log \frac{1}{r}}{k}\left\{\frac{1-\left(\frac{c}{n+c}\right)^{k}}{1-r^{n}}\right\} .
$$

Since $\lim _{n \rightarrow 0} \mu_{n}=1$ and $\lim _{n \rightarrow \infty} \mu_{n}=(-c \log r) / k, \mu$ cannot be totally monotone unless $-c \log r<k$. It is sufficient to show that there exists a positive integer $n$ such that $\mu_{n}<(-c \log r) / k$; i.e., $1-[c /(n+c)]^{k}<1-r^{n}$; i.e., $r^{n}(n+c)^{k}$ $<c^{k}$. But $c^{k}$ is a constant for each $k$, and $r^{n}(n+c)^{k} \rightarrow 0$ as $n \rightarrow \infty$. Therefore, for $n$ sufficiently large, $\mu_{n}<(-c \log r) / k$ and $\mu$ is not totally monotone.

Corollary 4.4. $\Gamma_{c}^{1}$ n.t.s. $H \sim \alpha_{2}(t, r)$. 
If $\Gamma_{c}^{1}$ t.s. $H \sim \alpha_{2}(t, r)$, then, using Theorem 4.1 leads to a contradiction of Theorem 4.5.

TheOREM 4.6. $C_{1}$ t.s. $H \sim \alpha_{s}(t, r)$.

Let $\mu(t)=(t+1)^{-1} \cdot(1-r)(t+1) /\left(1-r^{t+1}\right)=(1-r) \sum_{k=0}^{\infty} r^{(t+1) k}$. Each differentiated series of $\mu(t)$ is uniformly and absolutely convergent for $t \geqq 0$. Differentiating term by term, we obtain

$$
\mu^{(n)}(t)=(-1)^{n}(1-r)|\log r|^{n} \sum_{k=0}^{\infty} k^{n} r^{k(t+1)} .
$$

Since $\mu(0+)=\mu(0), \mu(t)$ is totally monotone.

CoROLlaRY 4.5. $H \sim \alpha_{3}(t, r)$ n.t.s. $H \sim \alpha_{1}(t, k, c), k=2,3, \cdots$.

If $H \sim \alpha_{3}(t, r)$ t.s. $H \sim \alpha_{1}(t, k, c)$, then using Theorem 4.6 leads to a contradiction of Theorem 4.2 (ii) and Corollary 4.1. See Theorem 4.8 (ii) for the case $k=1$.

COROLlaRY 4.6. $H \sim \alpha_{3}(t, r)$ n.t.s. $H \sim \alpha_{2}(t, r)$.

If $H \sim \alpha_{3}(t, r)$ t.s. $H \sim \alpha_{2}(t, r)$, then using Theorem 4.6 gives a contradiction to Theorem 4.4.

THEOREM 4.7. Let $r_{1} \neq r_{2}$.

(i) $H \sim \alpha_{3}\left(t, r_{1}\right)$ n.t.s. $H \sim \alpha_{3}\left(t, r_{2}\right)$.

(ii) $H \sim \alpha_{3}\left(t, r_{2}\right)$ n.t.s. $H \sim \alpha_{3}\left(t, r_{1}\right)$.

Proof of (i). Let $\mu(t)=\alpha_{3}\left(t, r_{1}\right) / \alpha_{3}\left(t, r_{2}\right)$. Then

$$
\mu(t)=\frac{\left(1-r_{2}\right)\left(1-r_{1}^{t+1}\right)}{\left(1-r_{1}\right)\left(1-r_{2}^{t+1}\right)} .
$$

Since $\lim _{t \rightarrow \infty} \mu(t)=\left(1-r_{2}\right) /\left(1-r_{1}\right), \mu(t)$ can be totally monotone only if $1-r_{2}<1-r_{1}$; i.e., $r_{1}<r_{2}$.

$$
\begin{aligned}
(-1) \mu^{\prime}(t)= & \frac{\left(1-r_{2}\right)}{\left(1-r_{1}\right)\left(1-r_{2}^{t+1}\right)^{2}}\left[r_{1}^{t+1}\left(1-r_{2}^{t+1}\right) \log r_{1}\right. \\
& \left.-r_{2}^{t+1}\left(1-r_{1}^{t+1}\right) \log r_{2}\right] .
\end{aligned}
$$

Since $r_{1}<r_{2}, r_{1}^{t+1}<r_{2}^{t+1}$ for $t>-1$. Hence $1-r_{1}^{t+1}>1-r_{2}^{t+1}$. Also $\log r_{1}$ $<\log r_{2}$. Therefore $(-1) \mu^{\prime}(t)<0$ for $t \geqq 0$. Therefore $\mu(t)$ is not totally monotone.

Proof of (ii). Because of the symmetry of $\mu(t)$ with respect to $r_{1}$ and $r_{2}$, $1 / \mu(t)$ is not totally monotone. 
THEOREM 4.8. (i) $\Gamma_{b}^{1}$ n.t.s. $H \sim \alpha_{3}(t, r)$.

(ii) $H \sim \alpha_{3}(t, r)$ n.t.s. $\Gamma_{b}^{1}$.

Proof of (i). Let $\mu_{n}=b(n+1)(1-r) /(n+b)\left(1-r^{n+1}\right) . \mu_{0}=1$ and $\mu_{n} \rightarrow b(1-r)$. If $b(1-r) \geqq 1, \mu$ is not totally monotone. Assume $b(1-r)<1$. If there exists a value of $n>0$ for which $\mu_{n}<b(1-r)$, then $\mu$ is not totally monotone. Assume $\mu_{n} \geqq b(1-r)$. Then

$$
\frac{b(n+1)(1-r)}{(n+b)\left(1-r^{n+1}\right)} \geqq b(1-r) ;
$$

i.e., $n+1 \geqq(n+b)\left(1-r^{n+1}\right)=n+b-(n+b) r^{n+1}$; i.e., $(n+b) r^{n+1} \geqq b-1$. But $b-1>0$ and $(n+b) r^{n+1} \rightarrow 0$. Therefore, for $n$ sufficiently large, $(n+b) r^{n+1}$ $<b-1$. Hence $\mu$ is not totally monotone.

To prove (ii) use the same technique as that used in the proof of Theorem 4.3.

TheOREM 4.9. For $1 \leqq k \leqq 4, H \sim \alpha_{1}(t, k+1, c)$ t.s. $H \sim \alpha_{1}(t, k, c)$.

Proof. Let

$$
\begin{aligned}
\mu_{k}(t) & =\frac{\alpha_{1}(t, k+1, c)}{\alpha_{1}(t, k, c)}=\frac{(k+1)^{-1}\left[1-c^{k+1}(t+c)^{-k-1}\right]}{\left(k^{-1}\right)\left[1-c^{k}(t+c)^{-k}\right]} \\
& =\frac{k}{(k+1)}\left\{\frac{1}{t / c+1}\right\}\left\{\frac{(t / c+1)^{k+1}-1}{(t / c+1)^{k}-1}\right\} \\
& =\frac{k}{k+1}\left\{1+\frac{t / c}{(t / c+1)\left[(t / c+1)^{k}-1\right]}\right\},
\end{aligned}
$$

(4.1) $\mu_{k}(l)=\frac{k}{k+1}\left\{1+\frac{1}{(t / c+1) \prod_{p=1}^{k-1}\left[(t / c)+2 \sin ^{2}(p \pi / k)-i \sin (2 p \pi / k)\right]}\right\}$;

where $(t / c+1)^{k}-1$ has been written in factored form, the common term of $t / c$ has been cancelled, and the value of the product is understood to be 1 for $k=1$. Then $\mu_{1}(t)=(1 / 2)\left[1+(t / c+1)^{-1}\right], \mu_{2}(t)=(2 / 3)\left[1+(t / c+1)^{-1}(t / c+2)^{-1}\right]$. and both are totally regular.

In (4.1) let the quantity in braces be represented by $1+\delta_{k}(t)$. Since $\delta_{k}(t)>0$, in order to show that $\mu_{k}(t)$ is totally regular for $k>2$, it is necessary and sufficient to show that $\delta_{k}(t)$ is totally monotone.

Let $\gamma_{0}(t)=(t / c+1)^{-1}, \quad \gamma_{p}(t)=\left(t / c+\alpha_{p}\right)^{-1}$, where $\alpha_{p}=2 \sin ^{2}(p \pi / k)$ $-i \sin (2 p \pi / k), 1 \leqq p \leqq k-1$. Note that $t / c+\alpha_{p}$ and $t / c+\alpha_{k-p}$ are complex conjugates, $1 \leqq p \leqq k-1$. For convenience restrict $p$ to $p>k / 2$. Then $\gamma_{0}^{(n)}(t)$ $=(-1)^{n} n ! c^{-n}(t / c+1)^{-n-1}$, and

$$
\gamma_{p}^{(n)}(l)=(-1)^{n} n ! c^{-n}\left(l / c+\alpha_{p}\right)^{-n-1}, \quad n=0,1,2, \cdots .
$$


Using Leibnitz's formula, De Moivre's Theorem, and the identity $\sum_{j-0}^{n} \sin (n-2 j) \theta \equiv 0$ for all real $\theta, n=0,1,2, \cdots$,

$$
D_{t}^{n}\left[\gamma_{p}^{(t)} \gamma_{k-p}^{(t)}\right]=\frac{(-1)^{n} n ! \sum_{j=0}^{n} \cos (n-2 j) \theta}{c^{n}\left|t / c+\alpha_{p}\right|^{n+2}},
$$

where $\theta=\arg \left(t / c+\alpha_{p}\right), k / 2 \leqq p \leqq k-1$.

Using the additional fact that, for $m$ an integer, $\sum_{j=0}^{n} \cos (n-2 j) \theta$ $=\sin (n+1) \theta / \sin \theta$ for $\theta \neq m \pi$, and is equal to $(-1)^{n m}(n+1)$ for $\theta=m \pi$,

$$
D_{i}^{n}\left[\gamma_{0}(t) \gamma_{p}(t) \gamma_{k-p}(t)\right]=\frac{(-1)^{n} n ! f(n, t, \theta)}{c^{n}(t / c+1)^{n+1}\left|t / c+\alpha_{p}\right|^{2}},
$$

where $f(n, t, \theta)=\left[\sum_{j=0}^{n}(t / c+1)^{j}\left|t / c+\alpha_{p}\right|^{-j} 2 \sinh i(j+1) \theta\right] / 2 i \sin \theta$.

By lengthy calculations it can be shown that $f(n, t, \theta)$ is positive for $t>0, n=1,2,3, \cdots$, provided $k \leqq 4$. Since $\delta_{3}(0+)=\delta_{3}(0), \delta_{3}(t)$ is totally monotone.

$\delta_{4}(t)=\gamma_{2}(t) \cdot\left[\gamma_{0}(t) \gamma_{1}(t) \gamma_{3}(t)\right]$. Since the quantity in brackets is totally monotone, and $\gamma_{2}(t)=(t / c+2)^{-1}$, which is totally monotone, $\delta_{4}(t)$ is totally monotone.

In order to attempt a proof of Theorem 4.9 for $k>4$ by this technique, the only hope lies in trying to show that $\gamma_{1}(t) \gamma_{2}(t) \gamma_{3}(t) \gamma_{4}(t)$ is totally monotone, or more generally, that $\prod_{p=k-2}^{k-1} \gamma_{p}(t) \gamma_{k-p}(t)$ is totally monotone. Otherwise, each new prime integer for $k$ presents a messy, prohibitive calculation, and does not lead to any sort of inductive formula. It can be shown that $\prod_{p=3}^{4} \gamma_{p}(t) \gamma_{5-p}(t)$ is not totally monotone.

THEOREM 4.10. $H \sim \alpha_{1}\left(t, k, c_{1}\right)$ t.s. $H \sim \alpha_{1}\left(t, k, c_{2}\right), c_{2}>c_{1}>0, k=1,2$.

For $k=1$ this theorem is a special case of R5. For $k=2$, a straightforward differentiation shows that $f(t)=\alpha_{1}\left(t, k, c_{1}\right) / \alpha_{1}\left(t, k, c_{2}\right)$ is totally monotone.

If $k>2$, the above differentiation becomes quite messy. However, I conjecture that Theorem 4.10 is true for $k \geqq 3$, and that Theorem 4.9 is true for $k \geqq 5$.

The following are open questions.

Is $H \sim \alpha_{2}(t, r)$ t.s. $H \sim \alpha_{3}(t, r)$ ?

Is $H \sim \alpha_{1}(t, k, b)$ t.s. $H \sim \alpha_{3}(t, r)$ ? (From Theorem 4.6 and Corollary 4.1 it is clear that $H \sim \alpha_{1}(t, k, a)$ t.s. $H \sim \alpha_{3}(t / r)$.)

5. Some equivalent methods.

THEOREM 5.1. Let $\mu_{k}=(a k+1) /(b k+1)$, where $a$ and $b$ are distinct nonzero complex numbers such that $a^{-1}$ and $b^{-1}$ are not negative integers. Then $\left(H_{\mu}\right)$ $=(I)$ iff $R(a)>0$ and $R(b)>0$.

The above theorem is stated in Hille [7, p. 422], and hence a proof is omitted. 
THEOREM 5.2. Let $\alpha$ be real, $R(a)>0$. Then $\left(\Gamma_{a}^{\alpha}\right)=\left(H^{\alpha}\right)$.

Because of the symmetry for positive and negative $\alpha$ we need consider only $\alpha \geqq 0$. The proof is trivial for $\alpha=0$, since the generating sequence is $\{1\}$. For a real, the result is stated in Hardy [6, p. 265].

Let $b=a^{-1}$. We need to show that $\left(H_{\lambda}^{\alpha}\right)=(I)$, where $\lambda_{k}=(b k+1) /(k+1)$. For $\alpha$ an integer the proof is trivial because $(H \dot{\lambda})=(I)$, by Theorem 5.1 and a finite product of such matrices yields a matrix equivalent to $I$.

Since $\lambda_{k}^{\alpha} / \lambda_{k}^{\alpha+1}=(k+1) /(b k+1)$, and using Hardy's argument, [6, p. 264, Theorem 211], we may assume $0<\alpha<1$.

Let $f(z)=(b+(1-b) z)^{\alpha}$ and $C=\{z|| z \mid \leqq 1\}$. Then $f(1)=1$, and a branch cut starting at $z_{0}=b /(b-1)$ can be defined so that $f(z)$ is analytic in $C$. $f$ has no zeros in $C$, since $\left|z_{0}\right|>1$. Note that $1 / f(z)$ is also analytic and has no zeros in C. From Hurwitz and Silverman [9, p. 3, Theorem 1], $\left(H_{\lambda}^{\alpha}\right)=(I)$.

Corollary 5.1. $\left(\Gamma_{a}^{\alpha}\right)=\left(C^{\alpha}\right)$ for all $\alpha>-1$.

Proof. $\left(C^{\alpha}\right)=\left(H^{\alpha}\right)$ for all $\alpha>-1$.

CoRollaRy 5.2. Let $\mu_{k}=(a k+1) /(b k+1), \quad R(a)>0$ and $R(b)>0$. Then $\left(H_{\mu}^{\alpha}\right)=(I)$ for all real $\alpha$.

Proof. By the symmetry of $\mu_{k}^{\alpha}$ it is evident that we need consider only $\alpha>0$. Note that

$$
\stackrel{\alpha}{\mu_{k}}=\frac{(a k+1)^{\alpha}}{(k+1)^{\alpha}} \cdot \frac{(k+1)^{\alpha}}{(b k+1)^{\alpha}}
$$

and each quantity on the right is equivalent to $I$ by Theorem 5.2.

COROLlary 5.3. $\Gamma_{a}^{\alpha}$ is regular for all $\alpha \geqq 0, R(a)>0$.

Proof. Using the $H_{\mu}$ of Corollary 5.2 with $a=1, b=a^{-1}, \Gamma_{a}^{\alpha}=H_{\mu}^{\alpha} \cdot H^{\alpha}$, and the product of two regular Hausdorff matrices is regular.

TheOREM 5.3. Let $\mu_{k}(\alpha)=\left[(k+a)^{\alpha}+b\right] / d(c k+1)^{\alpha}$, where $\alpha \geqq 0, \propto(a)>0$, $a(c)>0$, and $d=a^{\alpha}+b$. If $[a(a)]^{\alpha}>|b|$, then $\left(H_{\mu}\right)=(I)$. If $\alpha$ is a positive integer, then $R(a)>|b|^{1 / \alpha} \max _{0 \leq r<a}|\cos [(\theta+2 \pi r) / \alpha]|, \theta=\arg (-b)$, implies $\left(H_{\mu}\right)=(I)$, and this is best possible.

Proof. For $\alpha=0, \mu_{k}(0) \equiv 1$. Consider $\alpha>0$. Then $\mu_{k}(\alpha)=\beta_{k}(\alpha) \gamma_{k}^{\alpha}$, where $\beta_{k}(\alpha)=a^{\alpha}\left[(k+a)^{\alpha}+b\right] / d(k+a)^{\alpha}$, and $\gamma_{k}=(k+a) / a(c k+1)$.

By Corollary $5.2\left(H_{\gamma}^{\alpha}\right)=(I)$, and $H_{\beta}=d^{-1}\left[a^{\alpha} I+b H_{\lambda}^{\alpha}\right]$, where $\lambda_{k}=a /(k+a)$. Since $H_{\lambda}^{\alpha}$ is regular for each positive $\alpha$ by Corollary $5.3 H_{\beta}$ is regular, since $a^{\alpha}+b=d$.

It remains to prove $H_{\beta}^{-1}$ regular. To do this we appeal to Rogosinski, $[12$, p. 178] and the statement of 
PItT's Theorem. Let $T \sim \phi(t)$, where $\phi(t)$ is of regular bounded variation on $[0,1]$ (see Hille $[7$, p. 420]). Then $|T(z)| \geqq d>0, \mathfrak{R}(z) \geqq 0$, implies that $T^{-1}(z)$ is a Mellin transform, and thus $T$ is equivalent to $I$.

$T \sim \phi(t)$ is used to denote a regular Hausdorff method, with $\phi(t)$ the associated mass function; i.e., $\mu_{n}=\int_{0}^{1} t^{n} d \phi(t)$. The Mellin transforms of $\phi(t)$ are defined by $T(z)=\int_{0}^{1} t^{z} d \phi(t)$. Hence $\mu_{n}=T(n)$, and $T(z)$ is called the moment function of $T$. Let

$$
\beta_{\alpha}(z)=\frac{\left[a^{\alpha}(z+a)^{\alpha}+b\right]}{d(z+a)^{\alpha}}=\int_{0}^{1} t^{z} d \phi(t), \quad(R(z) \geqq 0),
$$

for some $\phi(t)$ of regular bounded variation (b.v.) on $[0,1]$. (See Rogosinski $\left[12\right.$, p. 167 , Theorem H.3].) Also $T(z)=\beta_{\alpha}(z)$,

$$
|T(z)|=\left|\frac{a^{\alpha}}{d}\left\{1+\frac{b}{(z+a)^{\alpha}}\right\}\right| \geqq\left|a^{\alpha} d^{-1}\right| \cdot|1-| b /(z+a)^{\alpha}|| .
$$

But $\left|(z+a)^{\alpha}\right|=\left[\left(x+a_{1}\right)^{2}+\left(y+b_{1}\right)^{2}\right]^{\alpha / 2}$, where $z=x+i y, a=a_{1}+i b_{1}$, and $|z+a|^{\alpha} \geqq|R(a)|^{\alpha}$, since $R(z) \geqq 0$ and $R(a)>0$.

Thus $|z+a|^{-\alpha} \leqq[R(a)]^{-\alpha}$ and $-|b| \cdot|z+a|^{-\alpha} \geqq-|b|[R(a)]^{-\alpha}$. Hence $|T(z)| \geqq\left|a^{\alpha} d^{-1}\right|\left[1-|b|[R(a)]^{-\alpha}\right]>0$, since $[R(a)]^{\alpha}>|b|$ by hypothesis.

Therefore $T^{-1}(z)$ is a Mellin transform and $T$ is equivalent to $I$; i.e., $\left(H_{\beta_{\alpha}}\right)=(I)$.

That $\left(H_{\beta_{\alpha}}\right)=(I)$ can also be proved using the technique of Hille and Tamarkin [8, p. 906, Theorem 5].

For $\alpha$ a positive integer,

$$
(k+a)^{\alpha}+b=\prod_{r=0}^{\alpha-1}\left\{k+a-|b|^{1 / \alpha} \operatorname{cis}[(\theta+2 r \pi) / \alpha]\right\},
$$

where $\theta=\arg (-b)$. Let $\lambda_{k}(r)=\left(k+c_{r}\right) / c_{r}(c k+1)$, where $c_{r}=a$ $-|b|^{1 / \alpha}$ cis $[(\theta+2 r \pi) / \alpha]$. Since $R(a)>|b|^{1 / \alpha} \cdot \max _{0 \leq r<a}|\cos [(\theta+2 r \pi) / \alpha]|$, $R\left(c_{r}\right)>0$, for $0 \leqq r<\alpha$. Also $R(c)>0$ by hypothesis. Therefore by Theorem $5.1\left(H_{\lambda}\right)=(I)$ for each $r$. Since $H_{\mu}=\prod_{r=0}^{\alpha-1} H_{\lambda(r)},\left(H_{\mu}\right)=(I)$.

To show that the theorem is best possible for $\alpha$ an integer, let $\alpha=1$, $a=1+i, b=-1, c=1$. Then $R(a)=|b| \cdot|\cos 0|, \mu_{k}=(k+i) / i(k+1)$, and by Theorem $5.1\left(H_{\mu}\right) \neq(I)$.

6. The method of summability. Let $R(a, b, \alpha)$ denote the matrix and the matrix method associated with the generating sequence $\xi_{k}=d\left[(k+a)^{\alpha}+b\right]^{-1}$, where $d=a^{\alpha}+b,[R(a)]^{\alpha}>|b|$.

Theorem 6.1. For each $\alpha \geqq 0, R(a, b, \alpha)$ as defined above, is regular and is equivalent to $C^{\alpha}$.

Proof. Trivial for $k=0$. Assume $k>0$. Using the $H_{\mu}$ of Theorem 5.3 with $c=1, R(a, b, \alpha)=H_{\mu}^{-1} \Gamma_{1}^{\alpha}=H_{\mu}^{-1} H^{\alpha}$. Therefore $R(a, b, \alpha)$ is regular. 
To prove the equivalence observe that $R(a, b, \alpha) \cdot H^{-\alpha}=H_{\mu}$. Thus $(R(a, b, \alpha))=\left(H^{\alpha}\right)=\left(\Gamma_{1}^{\alpha}\right)=\left(C^{\alpha}\right)$ by Corollary 5.1 .

Whenever the method $R(a, b, \alpha)$ is discussed, it will be assumed that $a$ and $b$ satisfy the conditions stated prior to Theorem 6.1 .

Corollary 6.1. If $0 \leqq \alpha<\beta$, then $(R(a, b, \alpha)) \subset(R(a, b, \beta))$, and with consistency.

Proof. Let $\mu_{k}=\left\{d^{\prime} /\left[(k+a)^{\beta}+b\right]\right\} \cdot\left\{\left[(k+a)^{\alpha}+b\right] d^{-1}\right\}$. Then $\mu_{k}=\lambda_{k} \gamma_{k} \nu_{k}$, where

$$
\lambda_{k}=\frac{d(k+a)^{\beta}}{a^{\beta}\left[(k+a)^{\beta}+b\right]}, \quad \gamma_{k}=\frac{\left[(k+a)^{\alpha}+b\right] a^{\alpha}}{d(k+a)^{\alpha}},
$$

and $\nu_{k}=(a /(k+a))^{\beta-\alpha}$. By Theorem 5.3 and Corollary 5.3, $H_{\lambda}, H_{\gamma}$, and $H_{\nu}$ are regular. Therefore $H_{\lambda}, H_{\gamma}, H_{\nu}=H_{\mu}$ is regular. All Hausdorff methods are consistent. (See Hardy [6, p. 262, footnote].)

COROLlARY 6.2. Let $\alpha \geqq 0, a, b, a^{\prime}, b^{\prime}$ be any numbers satisfying the conditions for regularity of $R(a, b, \alpha)$ and $R\left(a^{\prime}, b^{\prime}, \alpha\right)$. Then $(R(a, b, \alpha))=\left(R\left(a^{\prime}, b^{\prime}, \alpha\right)\right)$.

Proof. Note that $\mu_{k}=\beta_{k} \gamma_{k}$, where

$$
B_{k}=\frac{d(k+1)^{\alpha}}{(k+a)^{\alpha}+b}, \quad \gamma_{k}=\frac{\left(k+a^{\prime}\right)^{\alpha}+b^{\prime}}{d^{\prime}(k+1)^{\alpha}} ;
$$

then $H_{\beta}$ and $H_{\gamma}$ are equivalent to $I$ by Theorem 5.3.

7. Total comparison involving the $R(a, b, \alpha)$ method. In this section I shall examine the matrix method $R(a, b, \alpha)$, which was introduced in $\S 6$, with respect to total regularity, and compare its total relative strength with the $C^{\alpha}, H^{\alpha}$, and $\Gamma_{c}^{\alpha}$ methods, $c>0$. (Theorem 6.4 shows that the $R(a, b, \alpha)$ method is equivalent to $C^{\alpha}$, and hence to $H^{\alpha}$ and $\Gamma_{c}^{\alpha}$ for positive $c$.)

In discussing the $R(a, b, \alpha)$ method, it will be understood that $\alpha \geqq 0, a>0$, $a^{\alpha}>|b|, b$ is real, $d=a^{\alpha}+b$, and $\mu_{k}^{(\alpha)}=d\left[(k+a)^{\alpha}+b\right]^{-1}, k=0,1,2, \cdots$.

Also, for convenience, the following notation will be observed. $c$ and $f$ will denote arbitrary non-negative or positive numbers, $c^{\prime}$ and $f^{\prime}$ arbitrary nonpositive numbers. $a$ and $b$ will be restricted by $0<a<1, b>1$.

Theorem 7.1. For $\alpha \geqq 0, f>0, c^{\prime} \leqq 0, R\left(f, c^{\prime}, \alpha\right)$ is totally regular.

Proof. Trivial for $\alpha=0$, since $\mu_{k} \equiv 1$. Assume $\alpha>0$. Note that $R(f, 0, \alpha)$ $=\Gamma_{f}^{\alpha}$. Assume $c^{\prime}<0$. Let $c=-c^{\prime}$. Then $\mu_{k}^{(\alpha)}=d\left[(k+f)^{\alpha}-c\right]^{-1}$. Let $\mu(t)$ $=d\left[(t+f)^{\alpha}-c\right]^{-1}$. It will be sufficient to show that $\mu(t)$ is totally monotone, as $\mu_{k}^{(\alpha)}$ is regular by Theorem 6.1 .

$$
\mu(t)=d(t+f)^{-\alpha} \sum_{k=0}^{\infty} c^{k}(t+f)^{-\alpha k}=d \sum_{k=0}^{\infty} c^{k}(t+f)^{-\alpha k-\alpha},
$$


and, since $f^{\alpha}>\left|c^{\prime}\right|$, the series for $\mu(t)$ is uniformly and absolutely convergent for $t \geqq 0$. Since each differentiated series is uniformly convergent, we may differentiate term by term as often as we please and obtain $(-1)^{n} \mu^{(n)}(t)$ $=d \cdot s_{n}(t)$, where

$$
s_{n}(t)=\sum_{k=0}^{\infty} \frac{c^{k} \Gamma(\alpha k+\alpha+n)(t+f)^{-\alpha k-\alpha-n}}{\Gamma(\alpha k+\alpha)} .
$$

Since $s_{n}(t)$ consists entirely of non-negative terms, $\mu(t)$ is a totally monotone function.

For $c>0, R(f, c, \alpha)$ is not totally regular.

Counter-eXAmple. Let $\alpha=2$.

$$
\mu_{k}=d\left[(k+f)^{2}+c\right]^{-1}=d\left[\left(k+f+i c^{1 / 2}\right)\left(k+f-i c^{1 / 2}\right)^{-1} .\right.
$$

Let $\mu(t)=d /\left(t+\alpha_{1}\right)\left(t+\bar{\alpha}_{1}\right), \alpha_{1}=f+i c^{1 / 2}$. Use (4.2) with $k=2, c=1$, and the discussion which follows, to obtain

$$
\mu^{(n)}(t)=\frac{d(-1)^{n} n ! 2 \sin (n+1) \theta_{1}}{\left|t+\bar{\alpha}_{1}\right|\left|t+\alpha_{1}\right|^{n+1} \sin \theta_{1}},
$$

where $\theta_{1}=\operatorname{arc} \tan \left(c^{1 / 2}(t+f)^{-1}\right)$. By hypothesis, $f>c^{1 / 2}$. Therefore $0<\theta_{1}$ $<\pi / 4$. For all $t \geqq 0$, and each $n$ such that $(2 k-1) \pi<(n+1) \theta_{1}<2 k \pi$ for some integer $k,(-1)^{n} \mu^{(n)}(t)<0$. Therefore $\mu(t)$ is not totally monotone.

ThEOREM 7.2. If $\alpha \geqq 0, f>0, c \geqq 0$, then $\Gamma_{f}^{\alpha}$ t.s. $R(f, c, \alpha)$.

Proof. Cases for $\alpha=c=0$ are trivial. Assume $\alpha, c>0$. Let

$$
\mu_{n}=\frac{f^{\alpha}\left[(n+f)^{\alpha}+c\right]}{d(n+f)^{\alpha}}=f^{\alpha} d^{-1} \lambda_{n}+c d^{-1} \beta_{n},
$$

where $\lambda_{n} \equiv 1, \beta_{n}=f^{\alpha}(n+f)^{-\alpha}$. Since $\lambda$ and $\beta$ are clearly totally monotone, and $f^{\alpha}+\alpha=d, H \sim \mu$ is totally regular.

Theorem 7.3. If $\alpha>0, c^{\prime} \leqq 0$, then $R\left(f, c^{\prime}, \alpha\right)$ t.s. $\Gamma_{f}^{\alpha}$.

Proof. If $c^{\prime}=0$, the methods are identical. Assume $c^{\prime}<0$. Then $c^{\prime}=-c$ where $c>0$. Let $\mu_{n}=d(n+f)^{\alpha} / f^{\alpha}\left[(n+f)^{\alpha}-c\right]$. Let

$$
\mu(t)=\frac{d(t+f)^{\alpha}}{f^{\alpha}\left[(t+f)^{\alpha}-c\right]}=f^{-\alpha} d \sum_{k=0}^{\infty} c^{k}(t+f)^{-\alpha k} .
$$

Since the series and each derivative are uniformly and absolutely convergent for $t \geqq 0$,

$$
(-1)^{n} \mu^{(n)}(t)=f^{-\alpha} d \sum_{k=1}^{\infty} \frac{\Gamma(\alpha k+n) s_{n}(t)}{\Gamma(\alpha k)},
$$


where $s_{n}(t)=c^{k}(t+f)^{-\alpha k-n}, n=1,2,3, \cdots$. Therefore $\mu(t)$ is totally monotone.

Combining Theorems 7.2 and 7.3, and observing that $\Gamma_{1}^{\alpha}=H^{\alpha}$, we have

Corollary 7.1. Let $c^{\prime}\langle 0, c\rangle 0, \alpha>0$. Then $R\left(1, c^{\prime}, \alpha\right)$ t.s. $H^{\alpha}$ t.s. $R(1, c, \alpha)$.

TheOREM 7.4. For $c_{2}^{\prime}<c_{1}^{\prime}<0, R\left(f, c_{2}^{\prime}, \alpha\right)$ t.s. $R\left(f, c_{1}^{\prime}, \alpha\right)$.

Proof. Let $c_{1}^{\prime}=c_{2}^{\prime}+\delta, \delta>0, d_{i}=f^{\alpha}+c_{i}^{\prime}, i=1,2$.

$$
\begin{aligned}
\mu_{n} & =\frac{d_{2}}{d_{1}}\left\{\frac{(n+f)^{\alpha}+c_{1}^{\prime}}{(n+f)^{\alpha}+c_{2}^{\prime}}\right\}=\frac{d_{2}}{d_{1}}\left\{1+\frac{\delta}{(n+f)^{\alpha}+c_{2}^{\prime}}\right\} \\
& =\gamma+(1-\gamma) \beta_{n},
\end{aligned}
$$

where $\gamma=d_{2} / d_{1}, \beta_{n}=d_{2} /\left[(n+f)^{\alpha}+c_{2}^{\prime}\right]$. Since $H \sim \beta$ is totally regular by Theorem 7.1 , and $0<\gamma<1, H \sim \mu$ is totally regular.

TheOREM 7.5. $R\left(f_{2}, c^{\prime}, \alpha\right)$ t.s. $R\left(f_{1}, c^{\prime}, \alpha\right)$ for $f_{1}>f_{2}>0$.

Proof. Let

$$
\mu_{n}=\frac{d_{2}\left[\left(n+f_{1}\right)^{\alpha}+c^{\prime}\right]}{d_{1}\left[\left(n+f_{2}\right)^{\alpha}+c^{\prime}\right]}=d_{2} d_{1}^{-1}\left[1+\left(\lambda_{n}-\beta_{n}\right)\right],
$$

where $\lambda_{n}=\left(n+f_{1}\right)^{\alpha} /\left[\left(n+f_{2}\right)^{\alpha}+c^{\prime}\right], \beta_{n}=\left(n+f_{2}\right)^{\alpha} /\left[\left(n+f_{2}\right)^{\alpha}+c^{\prime}\right]$. By Theorem 7.3 $H \sim \lambda$ and $H \sim \beta$ are totally monotone. $\lambda_{n} \beta_{n}^{-1}=\left(n+f_{1}\right)^{\alpha} /\left(n+f_{2}\right)^{\alpha}$. Since $f_{1}>f_{2}, \lambda \beta^{-1}$ is totally monotone. To prove that $\lambda-\beta$ is totally monotone we use the following lemma.

LEMмA 7.1. Let $\alpha, \beta$ be real sequences, $\beta_{k} \neq 0$ for all $k$ and such that $\beta$ and $\alpha \beta^{-1}$ are totally monotone, with $\alpha_{k} \geqq \beta_{k}$ for $k=0,1,2, \cdots$. Then $\alpha-\beta$ is totally monotone.

Proof of Lemma. Let $\mu_{k}=\alpha_{k}-\beta_{k}$. For $n$ positive,

$$
\begin{aligned}
\Delta^{n}\left(\alpha_{k}-\beta_{k}\right) & =\Delta^{n}\left[\beta_{k}\left(\alpha_{k} \beta_{k}^{-1}-1\right)\right] \\
& =\sum_{j=0}^{n} C_{n, j} \Delta^{n-i} \beta_{k+j} \Delta^{j}\left(\alpha_{k} \beta_{k}^{-1}-1\right) \\
& =\Delta^{n} \beta_{k}\left(\alpha_{k} \beta_{k}^{-1}-1\right)+\sum_{j=1}^{n} C_{n, j} \Delta^{n-j} \beta_{k+j} \Delta^{j}\left(\alpha_{k} \beta_{k}^{-1}\right) .
\end{aligned}
$$

By hypothesis, $\beta$ and $\alpha \beta^{-1}$ are totally monotone and $\alpha_{k} \geqq \beta_{k}$. Therefore $\mu$ is totally monotone.

Returning to the proof of the theorem it is clear that $\lambda-\beta$, and hence $\mu$, are totally monotone.

For the next three theorems and the lemma, we shall use the following notation.

Let 


$$
\mu_{k}^{(\alpha)}=\frac{\left[(k+b)^{\alpha}+c^{\prime}\right] \Gamma(\alpha+1) \Gamma(k+1)}{d \Gamma(k+\alpha+1)},
$$

$\alpha$ an integer. Then $\mu_{k}^{(\alpha)}=\left[b^{\alpha} \nu_{k}^{(\alpha)}+c^{\prime} \gamma_{k}^{(\alpha)}\right] / d$, where $\nu_{k}^{(\alpha)}=\prod_{i=1}^{\alpha} \beta_{k}^{(i)}, \beta_{k}^{(1)}$ $=i(k+b) / b(k+i), \quad \gamma_{k}^{(\alpha)}=\Gamma(\alpha+1) \Gamma(k+1) / \Gamma(k+\alpha+1)$. Let $\lambda_{k}^{(\alpha)}=(k+\alpha)^{-1}$, $k+\alpha \neq 0$.

LemMA 7.2. $\Delta^{n} \gamma_{k}^{(\alpha)}=\alpha(1+\alpha) \cdots(n-1+\alpha) \gamma_{k}^{(\alpha)} \prod_{i=0}^{n-1} \lambda_{k+1+1}^{(\alpha)}, k=0,1,2$, $\cdots ; n=1,2,3, \cdots$.

Proof.

$$
\begin{aligned}
\Delta \gamma_{k}^{(\alpha)} & =\frac{\Gamma(\alpha+1) \Gamma(k+1)}{\Gamma(k+\alpha+1)}-\frac{\Gamma(\alpha+1) \Gamma(k+2)}{\Gamma(k+\alpha+2)} \\
& =\gamma_{k}^{(\alpha)}\left\{1-\frac{k+1}{k+1+\alpha}\right\} \\
& =\alpha \gamma_{k}^{(\alpha)} \lambda_{k+1}^{(\alpha)} .
\end{aligned}
$$

Assume the induction hypothesis.

$$
\begin{aligned}
\Delta_{\gamma_{k}}^{n+1} \gamma^{(\alpha)} & =\alpha(1+\alpha) \cdots(n-1+\alpha) \Delta \gamma_{k}^{(\alpha)} \prod_{i=0}^{n-1} \lambda_{k+i+1}^{(\alpha)} \\
& =\alpha(1+\alpha) \cdots(n-1+\alpha)\left[\gamma_{k}^{(\alpha)} \Delta \prod_{i=0}^{n-1} \lambda_{k+i+1}^{(\alpha)}+\prod_{i=0}^{n-1} \lambda_{k+i+2}^{(\alpha)} \Delta \gamma_{k}^{(\alpha)}\right] .
\end{aligned}
$$

Using the easily established identity

$$
\Delta\left(\prod_{i=0}^{n-1} \lambda_{k+i+1}^{(\alpha)}\right)=n \prod_{i=0}^{n} \lambda_{k+i+1}^{(\alpha)}
$$

and this lemma for $n=1$, we have

$$
\begin{aligned}
\Delta_{\gamma_{k}}^{n+1} \gamma_{k}^{(\alpha)} & =\alpha(1+\alpha) \cdots(n-1+\alpha)\left[\gamma_{k}^{(\alpha)} \cdot n \prod_{i=0}^{n} \lambda_{k+i+1}^{(\alpha)}+\prod_{i=1}^{n} \lambda_{k+i+1}^{(\alpha)} \alpha \gamma_{k}^{(\alpha)} \lambda_{k+1}^{(\alpha)}\right] \\
& =\alpha(1+\alpha) \cdots(n-1+\alpha)(n+\alpha) \gamma_{k}^{(\alpha)} \prod_{i=0}^{n} \lambda_{k+i+1}^{(\alpha)} .
\end{aligned}
$$

Lemma 7.2 also holds for complex $\alpha$, provided $\alpha \neq 0,-1,-2, \cdots$.

Theorem 7.6. For $b>1, c^{\prime}<0, C^{1}$ t.s. $R\left(b, c^{\prime}, 1\right)$ iff $b \geqq 1-c^{\prime}$.

Proof. $\mu_{k}^{(1)}=b \nu_{k}^{(1)} / d+c^{\prime} \gamma_{k}^{(1)} / d$. Noting that $\nu_{k}^{(1)}=\beta_{k}^{(1)}$ can be written as $(1 / b)+\left(1-b^{-1}\right) \lambda_{k}^{(1)}$, we have 


$$
\begin{aligned}
\Delta^{n} \mu_{k}^{(1)} & =\frac{1}{d}\left\{\frac{b\left(1-b^{-1}\right) n !}{\prod_{i=0}^{n}(k+i+1)}+\frac{c^{\prime} n !}{k+1} \prod_{i=0}^{n-1} \lambda_{k+i+1}^{(1)}\right\} \\
& =\frac{n !}{d} \prod_{i=0}^{n} \lambda_{k+1}^{(1)}\left[(b-1)+c^{\prime}\right] .
\end{aligned}
$$

Therefore $\left\{\mu_{k}^{(1)}\right\}$ is totally monotone iff $b \geqq 1-c^{\prime}$.

TheOREM 7.7. For $b>1, c^{\prime}<0, C^{2}$ t.s. $R\left(b, c^{\prime}, 2\right)$ iff $b>M$, where $M=\max \left\{3 / 2,1+\left(-c^{\prime}\right)^{1 / 2}\right\}$ and $M>\left(2-c^{\prime}\right)^{1 / 2}$.

Proof. $\mu_{k}^{(2)}=b^{2} \nu_{k}^{(2)} / d+c^{\prime} \gamma_{k}^{(2)} / d$. Using the formula for the $n$th difference of a product of two sequences, and again writing $\nu$ in terms of $\beta$ we have

$$
\begin{aligned}
& \Delta^{n} \nu_{k}^{(2)}= \sum_{j=0}^{n} C_{n, j} \Delta^{n-j} \beta_{k+j}^{(2)} \Delta^{j} \beta_{k}^{(1)} \\
&= \sum_{j=1}^{n-1} C_{n, j} \frac{(1-2 / b)(n-j) ! 2}{\prod_{i=0}^{n-j}(k+j+i+2)} \cdot \frac{(1-1 / b) j !}{\prod_{i=0}^{i}(k+i+1)}+\beta_{k}^{(1)} \Delta^{n} \beta_{k}^{(2)}+\beta_{k+n}^{(2)} \Delta^{n} \beta_{k}^{(1)} \\
&= \frac{2 n !(1-2 / b)(1-1 / b)}{\prod_{i=0}^{n+1}(k+i+1)} \sum_{j=1}^{n-1}(1)+\frac{2(k / b+1)(1-2 / b) n !}{\prod_{i=0}^{n}(k+i+2) \cdot(k+1)} \\
&+\frac{\left(\frac{k+n}{b}+1\right)(1-1 / b) n !}{\left(\frac{k+n}{2}+1\right) \prod_{i=0}^{n}(k+i+1)} \\
&= \frac{2 n !}{b^{2}} \prod_{i=0}^{n+1} \lambda_{k+i}^{(1)}[(b-2)(b-1)(n-1)+(k+b)(b-2) \\
& \Delta^{n} \mu_{k}^{(2)}= \frac{2 n !}{d} \prod_{i=0}^{n+1} \lambda_{k+i}^{(1)}[(b-2)(b-1)(n-1)+(k+b)(b-2) \\
&+(k+n+b)(b-1)] \\
&+\frac{c^{\prime}}{d} \frac{(n+1) ! \Gamma(3) \Gamma(k+1)}{\Gamma(k+3)} \prod_{i=0}^{n-1} \lambda_{k+i+1}^{(2)} \\
&= \frac{2 n !}{d} \prod_{i=0}^{n+1} \lambda_{k+i}^{(1)}[(b-2)(b-1)(n-1)+(k+b)(b-2) \\
&\left.+(k+n+b)(b-1)+c^{\prime}(n+1)\right] .
\end{aligned}
$$


Let

$$
\begin{aligned}
f(n, k, b)= & (n-1) b^{2}-3(n-1) b+2(n-1)+b^{2}+(k-2) b \\
& -2 k+(k+n) b+b^{2}-(k+n)-b+c^{\prime}(n+1) \\
= & (n+1) b^{2}+(2 k-2 n) b+n-3 k-2+c^{\prime}(n+1) .
\end{aligned}
$$

For each $n$, and $b \geqq 3 / 2, f \uparrow$ in $k$. For each $k$, and $b \geqq 1+\left(-c^{\prime}\right)^{1 / 2}, f \uparrow$ in $n$. Therefore, for $b \geqq M, f(n, k, b) \uparrow$ in $n$ and $k$. Hence $f(n, k, b) \geqq f(0,0, b)$ $=b^{2}-2+c^{\prime}$. Since $\mu_{k}^{(2)} \rightarrow 2 / d,\left\{\mu_{k}^{(2)}\right\}$ cannot possibly be totally monotone unless $d>2$; i.e., $b^{2}+c^{\prime}>2$; i.e., $b>\left(2-c^{\prime}\right)^{1 / 2}$. Therefore $f(0,0, b)>0$, and $\left\{\mu_{a}^{(2)}\right\}$ is totally monotone.

To show that the condition is necessary, there are three cases:

Case I. Assume $M=3 / 2$. For $b<3 / 2, f(1, k, b)=(2 b-3) k+2 b^{2}-2 b-1$ $+2 c^{\prime}$. If $a_{1}=2 b^{2}-2 b-1+2 c^{\prime} \leqq 0$, then $\Delta \mu_{k}^{(2)}<0$ for $k=1,2,3, \cdots$ If $a_{1}>0$, $\Delta \mu_{k}^{(2)}<0$ for $k \geqq\left[a_{1} /(3-2 b)\right]+1$.

Case II. Assume $M=1+\left(-c^{\prime}\right)^{1 / 2}$. Then for $b<M$,

$$
f(n, 0, b)=\left(b^{2}-2 b+1+c^{\prime}\right) n+b^{2}-2+c^{\prime} \text {. }
$$

Therefore $\Delta^{n} \mu_{0}^{(2)}<0$ for $n \geqq\left[\left(b^{2}-2+c^{\prime}\right) /\left(b^{2}-2 b+1+c^{\prime}\right)\right]+1$.

CASE III. For $M \leqq\left(2-c^{\prime}\right)^{1 / 2}$,

$$
\bar{\mu}=\lim \mu_{n} \geqq 1 \text {. }
$$

Therefore $\mu$ is not totally monotone.

TheOREM 7.8. For $b>1, c^{\prime}<0$,

$$
H^{2} \text { t.s. } R\left(b, c^{\prime}, 2\right) \text { iff } b \geqq 1+\left(-c^{\prime}\right)^{1 / 2} \text {. }
$$

Let $\mu_{k}^{(2)}=\left[(k+b)^{2}+c^{\prime}\right] / d(k+1)^{2}=b^{2}\left(\beta_{k}^{(1)}\right)^{2} / d+c^{\prime}\left(\gamma_{k}^{(1)}\right)^{2} / d$.

$$
\begin{aligned}
\Delta^{n}\left(\beta_{k}^{(1)}\right)^{2}= & \sum_{j=0}^{n} C_{n, j} \Delta^{n-j} \beta_{k+j}^{(1)} \Delta^{j} \beta_{k}^{(1)} \\
= & \sum_{j=1}^{n-1} C_{n, j}(1-1 / b)(n-j) ! \prod_{i=0}^{n-j} \lambda_{k+i+j}^{(1)} \\
& \times(1-1 / b) j ! \prod_{i=0}^{j} \lambda_{k+i}^{(1)}+\beta_{k}^{(1)} \Delta^{n} \beta_{k}^{(1)}+\beta_{k+n}^{(1)} \Delta^{n} \beta_{k}^{(1)} \\
= & n !(1-1 / b)^{2} \prod_{i=0}^{n} \lambda_{k+i}^{(1)} \sum_{j=1}^{n-1} \lambda_{k+j}^{(1)} \\
& +\left\{\frac{k+b}{b(k+1)}+\frac{k+n+b}{b(n+k+1)}\right\} n ! \prod_{i=0}^{n} \lambda_{k+i}^{(1)}(1-1 / b) .
\end{aligned}
$$




$$
\begin{aligned}
\Delta^{n}\left(\gamma_{k}^{(1)}\right)^{2}= & \Delta^{n}\left(\lambda_{k}^{(1)}\right)^{2}=\sum_{j=0}^{n} C_{n, j} \Delta^{n-j} \lambda_{k+j}^{(1)} \Delta^{j} \lambda_{k}^{(1)} \\
= & \sum_{j=0}^{n} C_{n, j}(n-j) ! \prod_{i=0}^{n-j} \lambda_{k+j+i}^{(1)} ! \prod_{i=0}^{j} \lambda_{k+i}^{(1)} \\
= & n ! \prod_{i=0}^{n} \lambda_{k+i}^{(1)} \sum_{j=0}^{n} \lambda_{k+j .}^{(1)} \\
\Delta^{n} \mu_{k}^{(2)}= & \frac{n ! \prod_{i=0}^{n} \lambda_{k+i}^{(1)}}{d}\left[(b-1)^{2} \sum_{j=1}^{n-1} \lambda_{k+j}^{(1)}\right. \\
& \left.+\left\{\frac{k+b}{k+1}+\frac{k+n+b}{n+k+1}\right\}(b-1)+c^{\prime} \sum_{j=0}^{n} \lambda_{k+j}^{(1)}\right], \\
& n ! \prod_{i=0}^{n} \lambda_{k+i}^{(1)} \\
& \left.+\frac{(k+b)(b-1)+c^{\prime}}{k+1}+\frac{(k+n+b)(b-1)+c^{\prime}}{n+k+1}\right\} .
\end{aligned}
$$

For $b \geqq 1+\left(-c^{\prime}\right)^{1 / 2},(b-1)^{2}+c^{\prime} \geqq 0$. Since $(k+n+b)(b-1)+c^{\prime}$ $>(k+b)(b-1)+c^{\prime} \geqq b(b-1)+c^{\prime},\left\{\mu_{k}^{(2)}\right\}$ is totally monotone if $b(b-1)+c^{\prime} \geqq 0$, which is true for $b \geqq\left(1+\left(1-4 c^{\prime}\right)^{1 / 2}\right) / 2$. But $\left(1+\left(1-4 c^{\prime}\right)^{1 / 2}\right) / 2<1+\left(-c^{\prime}\right)^{1 / 2}$ for all $c^{\prime}<0$. Therefore $\left\{\mu_{k}^{(2)}\right\}$ is totally monotone.

To prove the converse, assume $b<1+\left(-c^{\prime}\right)^{1 / 2}$ and fix $k$. The last two terms of (7.1) are bounded in $n . \sum_{j=1}^{n-1} \lambda_{k+j}^{(1)}$ is the sum of the first $n-1$ terms of a divergent series. Since $(b-1)^{2}+c^{\prime}<0$, the quantity in brackets will be negative for all $n$ sufficiently large. Therefore $\Delta^{n} \mu_{k}^{(2)}<0$ for all $n$ sufficiently large, and $\left\{\mu_{k}^{(2)}\right\}$ is not totally monotone.

A reasonable conjecture is that for $\alpha$ a positive integer, $H^{\alpha}$ t.s. $R\left(b, c^{\prime}, \alpha\right)$ iff $b \geqq 1+\left(-c^{\prime}\right)^{1 / \alpha}$.

Using Theorems 7.7, 7.8, and 2.3, it is a straightforward computation to show that, for $b>1, c^{\prime}<0, \alpha$ a positive integer, $H^{\alpha}$ t.s. $R\left(b, c^{\prime}, \alpha\right)$ for $b \geqq 1$ $+\left(-c^{\prime}\right)^{1 / \alpha}$.

Whether the condition on $b$ is best possible, for $\alpha>2$, is still an open question. To use the technique employed in Theorem 7.8 becomes intractible if continued further because of the complexity of the form of $\Delta^{n} \mu_{k}^{(\alpha)}$.

I shall now construct a table combining this last set of theorems with $\mathbf{R}(\mathrm{i})-\mathbf{R}$ (iii). The solid arrow replaces the letters t.s., with the arrow pointing to the weaker method. The dotted arrows represent unknown implications. No attempt is made to list unknown implications where partial results are 
available; e.g., Theorem 7.12 leaves open the question as to whether $R\left(1, c^{\prime}, \alpha\right)$ t.s. $\Gamma_{a}^{\alpha}$ for $d \leqq a$. This open question, along with others of the same type, has been omitted to prevent the table from becoming even more unwieldy.

For $0<\alpha<1,0<a<1, b<1, c^{\prime}<0, c>0$,

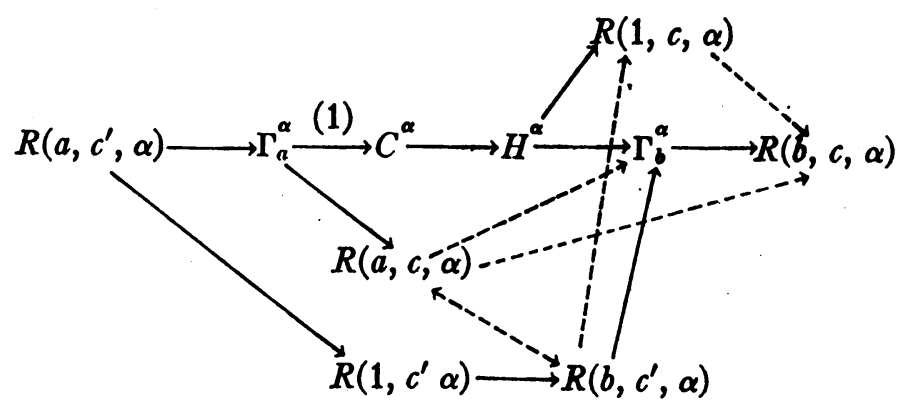

(1) For $a \leqq(\alpha+1) / 2$.

For $\alpha \geqq 1,0<a<1, b>1, c^{\prime}<0, c>0$,

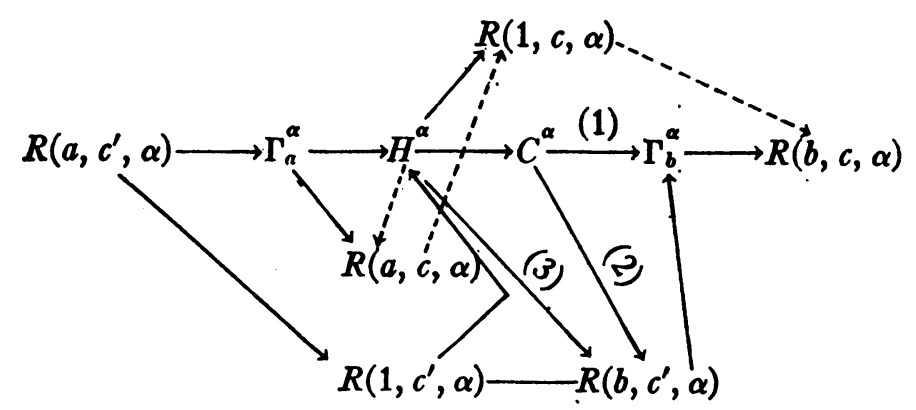

(1) For $b \geqq(\alpha+1) / 2$.

(2) For $\alpha=1, b \geqq 1-c^{\prime}$; for $\alpha=2, b \geqq M=\max \left\{3 / 2,1+\left(-c^{\prime}\right)^{1 / 2}\right\}$ and $M>\left(2-c^{\prime}\right)^{1 / 2}$. No results for $\alpha>2$.

(3) Proved for $\alpha$ an integer, $b \geqq 1+\left(-c^{\prime}\right)^{1 / \alpha}$.

The remainder of this section is taken up with negative results, to show that the above table is best possible in many instances. The obvious negative results will be omitted; for example, since $\Gamma_{b}^{\alpha}$ t.s. $R(b, c, \alpha)$, then clearly $R(b, c, \alpha)$ n.t.s. $\Gamma_{b}^{\alpha}$ from $[1$, p. 452$]$.

TheOREM 7.9. Let $\alpha>0, c>0, f_{2}>f_{1}>0$. Then $R\left(f_{2}, c, \alpha\right)$ n.t.s. $R\left(f_{1}, c, \alpha\right)$.

Proof. Let $\mu_{n}=d_{2}\left[\left(n+f_{1}\right)^{\alpha}+c\right] / d_{1}\left[\left(n+f_{2}\right)^{\alpha}+c\right]$, where $d_{i}=f_{i}^{\alpha}+c, i=1,2$. Since $f_{2}>f_{1}, d_{2}>d_{1}$. But $\mu_{n} \rightarrow d_{2} / d_{1}>1$. Therefore $\mu$ is not totally monotone.

This theorem shows us immediately that $R(b, c, \alpha)$ n.t.s. $R(1, c, \alpha)$ and that $R(1, c, \alpha)$ n.t.s. $R(a, c, \alpha)$. $c^{\prime}<0$.

Also, Theorem 7.9 is not a result of Theorem 7.4, since in Theorem 7.4 
Theorem 7.10. Let $\alpha>0, c, f,>0$. Then $R(f, c, \alpha)$ n.t.s. $C^{\alpha}$ for $\Gamma(\alpha+1)$ $\leqq f^{\alpha}+c$ and $C^{\alpha}$ n.t.s. $R(f, c, \alpha)$ for $f^{\alpha}+c \leqq \Gamma(\alpha+1)$.

Proof. Let $\mu_{n}=d \Gamma(n+\alpha+1) /\left[(n+f)^{\alpha}+c\right] \Gamma(n+1) \Gamma(\alpha+1) . \mu_{n} \rightarrow d / \Gamma(\alpha+1)$. Hence $\mu$ is not totally monotone for $d \geqq \Gamma(\alpha+1)$ and $\mu^{-1}$ is not totally monotone for $d \leqq \Gamma(\alpha+1)$.

Theorem 7.11. Let $0<\alpha<1, b>1, c^{\prime}<0$. (i) $H^{\alpha}$ n.t.s. $R\left(b, c^{\prime}, \alpha\right)$ and (ii) for $d>b^{\alpha-1}, R\left(b, c^{\prime}, \alpha\right)$ n.t.s. $H^{\alpha}$.

Let $\mu(t)=d^{-1}\left[(t+b)^{\alpha}+c^{\prime}\right](t+1)^{-\alpha}$.

$$
\begin{aligned}
\mu^{\prime}(t) & =\frac{1}{d}\left\{\frac{(t+1)^{\alpha} \alpha(t+b)^{\alpha-1}-\left[(t+b)^{\alpha}+c^{\prime}\right] \alpha(t+1)^{\alpha-1}}{(t+1)^{2 \alpha}}\right\} \\
& =\frac{\alpha}{d}\left\{\frac{t+1-(t+b)-c^{\prime}(t+b)^{1-\alpha}}{(t+1)^{1+\alpha}(t+b)^{1-\alpha}}\right\}, \\
(-1) \mu^{\prime}(t) & =\frac{\alpha}{d}\left\{\frac{b-1+c^{\prime}(t+b)^{1-\alpha}}{(t+1)^{1+\alpha}(t+b)^{1-\alpha}}\right\} .
\end{aligned}
$$

Proof of (i). Since $0<\alpha<1, c^{\prime}<0, b-1+c^{\prime}(t+b)^{1-\alpha}<0$ for all $t$ sufficiently large. Therefore $\mu(t)$ is not a totally monotone function.

Proof of (ii). Since $(-1) d / d t[1 / \mu(t)]=\mu^{\prime}(t)[\mu(t)]^{-2}, 1 / \mu(t)$ is not totally monotone if $\mu^{\prime}(t)<0$ for some $t \geqq 0$. $\mu^{\prime}(0)=1-b-c^{\prime} b^{1-\alpha} . \mu^{\prime}(0)<0$ for $b^{\alpha-1}$ $-b^{\alpha}-c^{\prime}<0$; i.e., $b^{\alpha-1}<b^{\alpha}+c^{\prime}=d$.

Theorem 7.12. Let $0<\alpha<1$. (i) $\Gamma_{a}^{\alpha}$ n.t.s. $R\left(1, c^{\prime}, \alpha\right)$. (ii) For $a<d$, $R\left(1, c^{\prime}, \alpha\right)$ n.t.s. $\Gamma_{a}^{\alpha}$.

Let $\mu(t)=a^{\alpha} d^{-1}\left[(t+1)^{\alpha}+c^{\prime}\right](t+a)^{-\alpha}$.

$$
\begin{aligned}
\mu^{\prime}(t) & =\frac{\alpha a^{\alpha}}{d}\left\{\frac{(t+a)-(t+1)^{1-\alpha}\left[(t+1)^{\alpha}+c^{\prime}\right]}{(t+a)^{1+\alpha}(t+1)^{1-\alpha}}\right\}, \\
(-1) \mu^{\prime}(t) & =\frac{\alpha a^{\alpha}}{d}\left\{\frac{1-a+c^{\prime}(t+1)^{1-\alpha}}{(t+a)^{1+\alpha}(t+1)^{1-\alpha}}\right\} .
\end{aligned}
$$

Proof of (i). For $t$ sufficiently large, $1-a+c^{\prime}(t+1)^{1-\alpha}<0$. Therefore $\mu(t)$ is not totally monotone.

Proof of (ii). $1 / \mu(t)$ will not be totally monotone if $\mu^{\prime}(t)<0 . \mu^{\prime}(0)=1-a+c^{\prime}$ $=d-a<0$ by hypothesis.

It is false that $R\left(1, c^{\prime}, \alpha\right)$ t.s. $\Gamma_{a}^{\alpha}$ for $d=a$.

Counter-EXAmPLE. Let $\alpha=a=d=1 / 2, c^{\prime}=-1 / 2$. Then $\mu_{n}^{-1}=(2 n+1)^{1 / 2}$ $/\left[2(n+1)^{1 / 2}-1\right] . \quad \mu_{0}^{-1}=1, \mu_{1}^{-1}=.947, \mu_{2}^{-1}=.645$. But $\mu_{n}^{-1} \rightarrow(2)^{1 / 2} / 2=.707$. Therefore $\mu^{-1}$ is not totally monotone.

Corollary 7.2. For $0<\alpha<1, c^{\prime}<0, C^{\alpha}$ n.t.s. $R\left(1, c^{\prime}, \alpha\right)$. 
Proof. Assume $C^{\alpha}$ t.s. $R\left(1, c^{\prime}, \alpha\right)$. Since $\Gamma_{\alpha}^{\alpha}$ t.s. $C^{\alpha}$ by $R(\mathrm{i}), \Gamma_{\alpha}^{\alpha}$ t.s. $R\left(1, c^{\prime}, \alpha\right)$, contradicting Theorem 7.12.

CoRollary 7.3. For $0<\alpha<1, R(1, c, \alpha)$ n.t.s. $R\left(b, c^{\prime}, \alpha\right)$.

Proof. Assume $R(1, c, \alpha)$ t.s. $R\left(b, c^{\prime}, \alpha\right) . H^{\alpha}$ t.s. $R(1, c, \alpha)$ by Corollary 7.1. Therefore $H^{\alpha}$ t.s. $R\left(b, c^{\prime}, \alpha\right)$, contradicting Theorem 7.11.

THEOREM 7.13. Let $0<\alpha<1, b>1, c>0$.

(i) $\Gamma_{b}^{\alpha}$ n.t.s. $R(1, c, \alpha)$ for $d<b$.

(ii) $R(1, c, \alpha)$ n.t.s. $\Gamma_{b}^{\alpha}$.

Proof of (i). Using the $\mu(t)$ of Theorem 7.12 with $a$ replaced by $b, c^{\prime}$ replaced by $c$,

$$
(-1) \mu^{\prime}(t)=\frac{\alpha b^{\alpha}}{d}\left\{\frac{1-b+c(t+1)^{1-\alpha}}{(t+b)^{1+\alpha}(t+1)^{1-\alpha}}\right\},
$$

$(-1) \mu^{\prime}(0)<0$ for $d<b$.

Proof of (ii). For $t$ sufficiently large, $\mu^{\prime}(t)<0$. Therefore $1 / \mu(t)$ is not totally monotone.

TheOREM 7.14. Let $0<\alpha<1,0<a<1, c>0$.

(i) $R(a, c, \alpha)$ n.t.s. $H^{\alpha}$.

(ii) $H^{\alpha}$ n.t.s. $R(a, c, \alpha)$ for $d<a^{\alpha-1}$.

Using the $\mu(t)$ of Theorem 7.11 with $b$ and $c^{\prime}$ replaced by $a$ and $c$,

$$
(-1) \mu^{\prime}(t)=\frac{\alpha}{d}\left\{\frac{a-1+c(t+a)^{1-\alpha}}{(t+1)^{1+\alpha}(t+\alpha)^{1-\alpha}}\right\} .
$$

Proof of (ii). $(-1) \mu^{\prime}(0)=\alpha d^{-1}\left(a-1+c a^{1-\alpha}\right)$, and is negative for $d<a^{\alpha-1}$.

Proof of (i). For $t$ sufficiently large, $1 / \mu(t)$ is not totally monotone.

Theorem 7.15. Let $\alpha \geqq 1$. Then $H^{\alpha}$ n.t.s. $R\left(b, c^{\prime}, \alpha\right)$ for $d<b^{\alpha-1}$.

Proof. Using the $\mu(t)$ of Theorem 7.11, $(-1) \mu^{\prime}(0)=\alpha d^{-1}\left(b^{\alpha}-b^{\alpha-1}+c^{\prime}\right)$ $=\alpha d^{-1}\left(d-b^{\alpha-1}\right)<0$. Therefore $\mu(t)$ is not totally monotone.

Theorem 7.16. Let $\alpha \geqq 1, \alpha$ an integer. Then $C^{\alpha}$ n.t.s. $R\left(b, c^{\prime}, \alpha\right)$ for $b<b_{0}$, where $b_{0}$ is the positive root of $(\alpha+1) b^{\alpha}-(b+1)^{\alpha}+\alpha c^{\prime}=0$.

Proof. Let $\mu_{n}=\left[(n+b)^{\alpha}+c^{\prime}\right] \Gamma(\alpha+1) \Gamma(n+1) / d \Gamma(n+\alpha+1)$.

$$
\Delta \mu_{n}=\mu_{n}\left\{1-\frac{(n+1)\left[(n+b+1)^{\alpha}+c^{\prime}\right]}{(n+\alpha+1)\left[(n+b)^{\alpha}+c^{\prime}\right]}\right\} .
$$

Let $f(t, b)=(t+\alpha+1)\left[(t+b)^{\alpha}+c^{\prime}\right]-(t+1)\left[(t+b+1)^{\alpha}+c^{\prime}\right] . f(0, b)$ $=(\alpha+1)\left(b^{\alpha}+c^{\prime}\right)-(b+1)^{\alpha}-c^{\prime}=(\alpha+1) b^{\alpha}-(b+1)^{\alpha}+\alpha c^{\prime}=\alpha b^{\alpha}-\sum_{k=1}^{\alpha-1} C_{\alpha, k} b^{k}$ $-1+\alpha c^{\prime} . f(0, b)=0$ has one real, positive root. Call it $b_{0}$. Note that $f(0,0)<0$. 
Then for $b<b_{0}, f(0, b)<0$. Therefore $\Delta \mu_{0}<0$. Therefore $\mu$ is not totally monotone.

8. Linear combinations of Hausdorff matrices. Vanderburg in [13] proves, as two of his results, the following:

(I) At least when they are both defined and are real and regular, the methods $H^{\alpha+1},\left[C^{\alpha}-\Gamma(\alpha+1) H^{\alpha}\right][1-\Gamma(\alpha+1)]^{-1}$ are equivalent.

(II) At least, if $\alpha, \beta, \alpha+\beta$ are real and exceed -1 , and neither $\alpha$ nor $\beta$ is zero, then the summability method

$$
\begin{aligned}
{\left[\Gamma(\alpha+\beta+1) C^{\alpha} C^{\beta}-\Gamma(\alpha+1) \Gamma(\beta+1) C^{\alpha+\beta}\right] } \\
\cdot[\Gamma(\alpha+\beta+1)-\Gamma(\alpha+1) \Gamma(\beta+1)]^{-1}
\end{aligned}
$$

is equivalent to $C^{\alpha+\beta+1}$.

It is the purpose of this section to employ the principle underlying (I) and (II) to a wider class of Hausdorff matrices.

The notation of [13] will be used throughout this section, and the reader is referred there for definitions of special symbols.

Let $K$ denote any regular Hausdorff matrix. Let $k$ denote the normalizing factor of $K$. By normalizing factor is meant the constant part of the generating sequence which makes $\mu_{0}=1$. For example the normalizing factors for the $H^{\alpha}, C^{\alpha}, \Gamma_{a}^{\alpha}$, and $R(c, b, \alpha)$ matrices are $1, \Gamma(\alpha+1), a^{\alpha}$, and $d=c^{\alpha}+b$, respectively.

The essential idea in (I) and (II) is the following. Each of two Hausdorff matrices, equivalent to $H^{\alpha}$, is divided by its normalizing factor, and then one matrix is subtracted from the other. The difference matrix is then divided by the difference of the normalizing factors, giving rise to a matrix which is equivalent, under appropriate restrictions, to $H^{\alpha+1}$. A formalization of these ideas, in one direction, is embodied in the following theorem.

THEOREM 8.1. Let $K_{1}^{\alpha}, K_{2}^{\alpha}$ denote any Hausdorff matrices equivalent to $H^{\alpha}$, $K_{1}^{\alpha}(z)$ and $K_{2}^{\alpha}(z)$ the associated Mellin transforms, and $k_{1}$ and $k_{2}$ the respective normalizing factors, $k_{1} \neq k_{2}$. If, for each $\alpha$ with $R(\alpha)>-1$, there exists an $\epsilon, 0<\epsilon$ $<1$, such that

$$
\frac{K_{1}^{\alpha}(z)}{k_{1}}-\frac{K_{2}^{\alpha}(z)}{k_{2}}=c(z+1)^{-\alpha-1}+O\left(|z+1|^{-\alpha-2}\right)
$$

uniformly on $R_{\epsilon}=\{z \mid R(z)>-\epsilon\}$, where $c$ is a constant, then

$$
\left[k_{2}^{\alpha} K_{1}^{\alpha}-k_{1}^{\alpha} K_{2}^{\alpha}\right]\left(k_{2}^{\alpha}-k_{1}^{\alpha}\right)^{-1} \supset H^{\alpha+1} \text {. }
$$

Let

$$
T(z)=\left[k_{1}^{-1} K_{1}^{\alpha}(z)-k_{2}^{-1} K_{2}^{\alpha}(z)\right](z+1)^{\alpha+1} .
$$

From $\left(^{*}\right), T(z)$ is clearly a Mellin transform. 
To give an indication of the applicability of Theorem 8.1, other than to the proof of one of the inclusions of (I) and (II), the following inclusions are listed. All of the inclusions are expressed in terms of $C^{\alpha+1}$ for uniformity only. They could have been expressed in terms of any other equivalent Hausdorff matrix.

Unless otherwise specified, it will be understood that $a, b, c$, and $\alpha$ are real, that $b$ and $c$ are such that $R(a, b, \alpha)$ is regular, and that the differences between normalizing factors are not zero.

(i) $\left[a^{\alpha} C^{\alpha}-\Gamma(\alpha+1) \Gamma_{a}^{\alpha}\right]\left[a^{\alpha}-\Gamma(\alpha+1)\right]^{-1} \supset C^{\alpha+1}$, at least for $a>0, \alpha>-1$.

(ii) $\left[a^{\alpha} H^{\alpha}-\Gamma_{a}^{\alpha}\right]\left(a^{\alpha}-1\right)^{-1} \supset C^{\alpha+1}$, at least for $a>0, \alpha>-1$.

(iii) $\left[d C^{\alpha}-\Gamma(\alpha+1) R(c, b, \alpha)\right][d-\Gamma(\alpha+1)]^{-1} \supset C^{\alpha+1}$, at least for $\alpha \geqq 1$.

(iv) $\left[d H^{\alpha}-R(c, b, \alpha)\right]\left(d-a^{\alpha}\right)^{-1} \supset C^{\alpha+1}$, at least for $\alpha \geqq 1$.

(v) $\left[d \Gamma_{a}^{\alpha}-a^{\alpha} R(c, b, \alpha)\right]\left(d-a^{\alpha}\right)^{-1} \supset C^{\alpha+1}$, at least for $\alpha \geqq 1$.

(vi) $\left[d_{2} R\left(c_{1}, b_{1}, \alpha\right)-d_{1} R\left(c_{2}, b_{2}, \alpha\right)\right]\left(d_{2}-d_{1}\right)^{-1} \supset C^{\alpha+1}$, at least for $\alpha \geqq 1$.

(vii) $\left[b^{\alpha} \Gamma_{a}^{\alpha}-a^{\alpha} \Gamma_{b}^{\alpha}\right]\left(b^{\alpha}-a^{\alpha}\right)^{-1} \supset C^{\alpha+1}$, at least for $\alpha>-1, a>0$.

(viii) $\left[C^{\alpha} C^{\beta}-\Gamma(\alpha+1) \Gamma(\beta+1) H^{\alpha+\beta}\right][1-\Gamma(\alpha+1) \Gamma(\beta+1)]^{-1} \supset C^{\alpha+\beta+1}$, at least for $\alpha, \beta, \alpha+\beta>-1$.

(ix) $\left[a^{\alpha+\beta} C^{\alpha} C^{\beta}-\Gamma(\alpha+1) \Gamma(\beta+1) \Gamma_{a}^{\alpha+\beta}\right]\left[a^{\alpha+\beta}-\Gamma(\alpha+1) \Gamma(\beta+1)\right]^{-1} \supset C^{\alpha+\beta+1}$, at least for $\alpha, \beta, \alpha+\beta>-1, a>0$.

(x) $\left[d C^{\alpha} C^{\beta}-\Gamma(\alpha+1) \Gamma(\beta+1) R(c, b, \alpha+\beta)\right][d-\Gamma(\alpha+1) \Gamma(\beta+1)]^{-1} \supset C^{\alpha+\beta+1}$, at least for $\alpha, \beta>-1, \alpha+\beta \geqq 1$.

Since all of the above are proved similarly, I shall prove (i) only.

Let

$$
T(z)=\frac{\Gamma(z+1)}{\Gamma(z+\alpha+1)}-\frac{1}{(z+a)^{\alpha}} .
$$

From $[13,(1.9)]$,

$$
T(z)=\frac{1}{(z+1)^{\alpha}}+\frac{\alpha(\alpha-1)}{2(z+1)^{\alpha+1}}+O\left(|z+1|^{-\alpha-2}\right)-\frac{1}{(z+a)^{\alpha}} .
$$

CASE I. $a \geqq 1$.

$$
\begin{aligned}
\frac{1}{(z+1)^{\alpha}}-\frac{1}{(z+a)^{\alpha}} & =(z+1)^{-\alpha}\left\{1-\left(\frac{z+1}{z+a}\right)^{\alpha}\right\} \\
& =(z+1)^{-\alpha}\left\{1-\left(1-\frac{a-1}{z+a}\right)^{\alpha}\right\} \\
& =(z+1)^{-\alpha}\left[\alpha(a-1)(z+a)^{-1}+O\left(|z+a|^{-2}\right) .\right.
\end{aligned}
$$

It is clear that an $\epsilon$ can be found so that, on $R_{e}$, the above series expansion remains valid, and $\left|(z+1)(z+a)^{-1}\right|$ is uniformly bounded. Substituting (8.3) into $T_{1}(z)$ then shows that $T_{1}(z)$ satisfies $\left({ }^{*}\right)$ of Theorem 8.1.

CASE II. For $0<a<1$, use the Laurent expansion for (8.2) in powers of 
$z+1$, which will be valid everywhere outside the circle $|z+1|=1-a$. The remainder of the proof is the same as Case $I$.

For $\alpha$ a positive integer, the above results hold also for $R(a)>0,[R(c)]^{\alpha}$ $>|b|,\left[R\left(c_{i}\right)\right]^{\alpha}>\left|b_{i}\right|, i=1,2$.

To prove the opposite inclusion, or the converses, of (i)-(x), it is necessary and sufficient to show that condition (B) of $[13$, p. 467$]$ is satisfied; i.e., that each Mellin transform does not vanish on $R_{0}$. The converses pose a much more difficult problem. Some partial results are listed below. For brevity only the restrictions on the parameters are listed.

C(i) At least for $a>0, \alpha>-1, \alpha \neq 0, \alpha_{0}$, where $\alpha_{0}$ will be defined in the proof.

C(ii) At least for $a>0, \alpha>-1, \alpha \neq 0$. For complex $a$, the converse remains true for nonzero $\alpha>-1$, provided $R(a) \geqq 1$.

C(iv) At least for $\alpha \geqq 0, b>0, \Omega(c) \geqq 1$.

$\mathrm{C}$ (v) At least for $\alpha \geqq 0, a, b, c$ real $, b>0, c \geqq a$.

$\mathrm{C}$ (vi) At least for $\alpha \geqq 0, b_{i}, c_{i}$ real, $i=1,2$, and $c_{2} \geqq c_{1}, b_{2} \geqq b_{1}$.

$\mathrm{C}$ (vii) At least for $\alpha>0, a, b$ real, $a \neq b$, or $\mathcal{R}(a)>\mathscr{A}(b), g(a)>g(b)$, or with the directions of the inequalities reversed.

$\mathrm{C}$ (viii) Since the matrix is symmetric in $\alpha$ and $\beta$, there is no loss in assuming $\alpha \leqq \beta$. Under this convention, the converse is true at least for (A) $\alpha \geqq 1$; (B) $0 \leqq \alpha$ $<1,0<\beta<1$; (C) $-1<\alpha<0, \beta \leqq 0$ or $\beta \geqq 1$.

$\mathrm{C}$ (ix) With $\alpha \leqq \beta$, the converse holds at least for (A) $\alpha \geqq \alpha_{0}$; (B) $-1<\alpha<0$, $\beta \leqq 0$, or $\beta \geqq \alpha_{0}$; and $(\mathrm{C}) 0 \leqq \alpha<\alpha_{0}, 0<\beta<\alpha_{0}$, for the $\alpha_{0}$ of $\mathrm{C}(\mathrm{i})$.

Proof of C(i). From (8.1), $T_{1}(z)=0$ if and only if $F_{1}(z, \alpha)=1$, where

$$
F_{1}(z, \alpha)=\frac{\Gamma(z+\alpha+1)}{(z+a)^{\alpha} \Gamma(z+1)} .
$$

For real $a$ and $z$ it can be shown, as in [13], that $F_{1}(\alpha+x+1)$ is a logarithmically convex function of $\alpha$. Hence it cannot take on the value 1 more than twice - once at $\alpha=0$ and again for some positive value $\alpha_{0}$, depending on $a$.

For $z=x+i y, y \neq 0, R(a)>0$, and using the procedure of [13], we obtain

$$
2 \log \left|F_{1}(z, \alpha)\right|=\sum_{j=0}^{\infty}\left\{\int_{x+j+\mathbb{R}(a)}^{x+j+\mathbb{R}^{(a)+1}} \frac{2 t d t}{t^{2}+(y+g(a))^{2}}-\int_{x+j+1}^{\alpha+x+j+1} \frac{2 t d t}{t^{2}+v^{2}} .\right.
$$

For property (a) of $[13$, p. 469$]$, we observe that

$$
\begin{aligned}
& 2\left[\log \left|F_{1}(z,-x,-1)\right|\right.\left.-\log \left|F_{1}(z,-x)\right|\right] \\
&=\sum_{j=0}^{\infty}\left\{\int_{j}^{j+1} \frac{2 t d t}{t^{2}+y^{2}}-\int_{x+j+}^{x+j+\Re_{(a)}^{(a)+1}} \frac{2 t d t}{t^{2}+(y+g(a))^{2}}\right\} .
\end{aligned}
$$

For $a$ real, property (a) is established by using the following lemma.

LemMa 8.1. If $f(t)$ is a function defined on $(0, \infty)$ which is positive, continuous, and monotone decreasing in $(0, \infty)$, then for $c>0$ and $0<x_{1}<x_{2}$, 


$$
\int_{x_{1}}^{x_{1}+c} f(t) d t-\int_{x_{2}}^{x_{2}+z} f(t) d t>0 .
$$

If $a$ is complex, and $|y+g(a)| \geqq|y|$, then property (a) is still clearly satisfied. Those values of $z$ for which $|y+g(a)|<|y|$ remain an open question.

Next we observe that

$$
\begin{aligned}
& 2 \frac{\partial}{\partial \alpha} \log \left|F_{1}(z, \alpha)\right| \\
& \quad=\sum_{j=0}^{\infty}\left\{\int_{x+j+R(a)}^{x+j+R(a)+1} \frac{2 t d t}{t^{2}+(y+g(a))^{2}}-\frac{2(\alpha+x+j+1)}{(\alpha+x+j+1)^{2}+y^{2}}\right\} .
\end{aligned}
$$

Hence, property (b) is also satisfied.

Finally we observe that $\partial^{2}\left(\log \left|F_{1}(z, \alpha)\right|\right) / \partial \alpha^{2}$ is identical to that of the $F$ in [2]. Therefore statements (c) and (d) automatically hold. [13].

At this point I should like to point out several typographical errors of

On page 469 , formula $(2.6)$, the quantity $[(z+\alpha+1) /(z+1)]$ should have an exponent of -1 .

In formula (2.9), the lower limit on the first integral should be $x+j+1$.

Formula (2.11) is the third partial derivative, provided the right-hand side is multiplied by 2 .

In formula (3.7), page 471 , the first term in the numerator should read $(\alpha+\beta+z+j+1)$.

In formula (4.13), page 473, there should be a plus sign between $\alpha / \mu^{2}$ and the summation sign.

Proofs of the other converses are straightforward and will be omitted.

I conjecture that Theorem 8.1 is true for all regular Hausdorff matrices equivalent to $C^{\alpha}$, and possessing different normalizing factors.

Finally, I am indebted to $\mathrm{K}$. Zeller and A. Wilansky for making me aware of Vanderburg's paper.

\section{REFERENCES}

1. S. K. Basu, On the total relative strength of the Hölder and Cesaro methods, Proc. London Math. Soc. vol. 50 (1948-1949) pp. 447-462.

2. - On hypergeometric summability involving infinite limits, Proc. Amer. Math. Soc vol. 5 (1954) pp. 226-238.

3. - On the total relative strength of some Hausdorff methods equivalent to the identity, Amer. J. Math. vol. 76 (1954) pp. 389-398.

4. - On comparison of the total strength of some Hausdorff methods, Math. Z. vol. 67 (1957) pp. 303-309.

5. H. J. Greenberg and H. S. Wall, Hausdorf means included between $(C, 0)$ and $(C, 1)$, Bull. Amer. Math. Soc. vol. 48 (1942) pp. 774-783.

6. G. H. Hardy, Divergent series, Oxford, 1949. 
7. E. Hille, Functional analysis and semi-groups, Amer. Math. Soc. Colloquium Publications, vol. 31, 1948.

8. E. Hille and J. D. Tamarkin, On moment functions, Proc. Nat. Acad. Sci. U.S.A. vol. 19 (1933) pp. 902-908.

9. W. A. Hurwitz and L. L. Silverman, On the consistence and equivalence of certain definitions of summability, Trans. Amer. Math. Soc. vol. 18 (1917) pp. 1-20.

10. B. E. Rhoades, Total comparison between two Cesdro matrices, Abstract 553-96, Notices Amer. Math. Soc. vol. 7 (1958) p. 828.

11. - Total comparison among some totally regular Hausdorff methods, Math. Z. vol. 72 (1960) pp. 463-466.

12. W. W. Rogosinski, On Hausdorff's method of summability, Proc. Cambridge Philos. Soc. vol. 38 (1942) pp. 166-192.

13. B. Vanderburg, Certain linear combinations of Hausdorff summability methods, Trans. Amer. Math. Soc. vol. 71 (1951) pp. 466-477.

14. A. Wilansky, An application of Banach linear functionals to summability, Trans. Amer. Math. Soc. vol. 67 (1949) pp. 59-68.

Lafayette College,

Easton, Pennsylvania 